\title{
Analytical Solution to Equivalent Consumption Minimization Strategy for Series Hybrid Electric Vehicles
}

Iman Shafikhani and Jan Åslund

The self-archived postprint version of this journal article is available at Linköping University Institutional Repository (DiVA):

http://urn.kb.se/resolve?urn=urn:nbn:se:liu:diva-173452

N.B.: When citing this work, cite the original publication.

Shafikhani, I., Åslund, J., (2021), Analytical Solution to Equivalent Consumption Minimization Strategy for Series Hybrid Electric Vehicles, IEEE Transactions on Vehicular Technology.

https://doi.org/10.1109/TVT.2021.3059205

Original publication available at:

https://doi.org/10.1109/TVT.2021.3059205

Copyright: Institute of Electrical and Electronics Engineers

http://www.ieee.org/index.html 


\title{
Analytical Solution to Equivalent Consumption Minimization Strategy for Series Hybrid Electric Vehicles
}

\author{
Iman Shafikhani, Jan Åslund
}

\begin{abstract}
Analytical optimal solution to the energy management of hybrid electric vehicles is of interest from theoretical and practical perspectives. Particularly, effort has been made to derive analytical solution to the energy management problem for series hybrid electric vehicles using Pontryagin's minimum principle (PMP). However, admissibility of the system input was not fully explored in determining the optimal input candidates. In this paper, the analytical solution for the same problem is found by partitioning the positive power demand set into four subsets, where the solution is derived for each case separately according to the corresponding admissible input set. The analytical solution is verified through comparison with numerical solution for a series hybrid electric wheel loader, and two different drive cycles are considered for this purpose. From the proposed solution, effective equivalence factor bounds are found and used to construct an adaptive equivalent consumption minimization strategy. The proposed strategy and the analytical solution are implemented together for the same vehicle to demonstrate their effectiveness in dealing with real-world applications. Simulations are performed for 12 drive cycles, and the results are compared to the ones achieved by PMP-based optimal control where the optimization is done numerically. Simulation results suggest that the proposed methodology is relatively fast and has satisfactory performance in presence of drive cycle uncertainty. It is observed that the proposed method fulfills charge sustenance, and the achieved fuel consumption figures are very close to the optimal benchmarks found by the non-causal method.
\end{abstract}

Index Terms-Energy management, Equivalent consumption minimization strategy (ECMS), Pontryagin's minimum principle (PMP), Hybrid electric vehicle (HEV).

\section{NOMENCLATURE}

$\gamma \quad$ Slope of the line that approximates genset's static map for positive electric power of the genset.

$\hat{\lambda}_{\max } \quad$ Estimate of maximum equivalence factor.

$\hat{\lambda}_{\text {min }}$ Estimate of minimum equivalence factor.

$\lambda \quad$ Equivalence factor.

$\lambda_{\max }^{e f f} \quad$ Maximum effective equivalence factor, above which the optimal solution does not change.

$\lambda_{\min }^{e f f} \quad$ Minimum effective equivalence factor, below which the optimal solution does not change.

$\lambda_{c y c}^{\text {nom }}$ Equivalence factor corresponding to nominal drive cycle.

$\mathcal{P}_{d} \quad$ Sequence of sampled power requests.

$\mathcal{P}_{d}^{\delta} \quad$ Sequence of sampled power requests of non-ideal drive cycle.

I. Shafikhani and J. Åslund are with the Vehicular Systems Group, Department of Electrical Engineering, Linköping University, Linköping, 58183 Sweden, e-mail: (iman.shafikhani@liu.se).
$\mathcal{U}_{t} \quad$ Admissible input set at time $t$.

$\mathcal{X} \quad$ Admissible state set.

$\mu \quad$ Costate vector in the Hamiltonian, corresponding to the local state constraint.

$\omega \quad$ Genset's shaft speed.

$P_{b, \max }$ Maximum battery power at the terminal.

$P_{b, \text { nom }}$ Nominal battery power.

$P_{g s, \max }$ Maximum electric power that the genset can generate.

$\stackrel{+}{\mathcal{P}}_{d} \quad$ Set of positive sampled power demands, which is a subset of $\mathcal{P}_{d}$.

$\mathrm{SOC}_{H}$ Upper limit of the local constraint for SOC.

$\mathrm{SOC}_{L}$ Lower limit of the local constraint for SOC.

$f \quad$ System dynamics.

$f_{\lambda} \quad$ A function that computes $\lambda$ for a given $P_{c r}$.

$H \quad$ Hamiltonian function.

$I_{b} \quad$ Battery current.

$J_{e} \quad$ Genset's shaft inertia.

$J_{f} \quad$ Cost function to be minimized for energy management (equal to total mass of fuel consumed in a driving mission).

$J_{P M P}$ General formulation of the cost function for optimal control.

$m_{f} \quad$ Mass of the fuel consumed.

$N_{c y c} \quad$ Number of elements in $\mathcal{P}_{d}$.

$p \quad$ Costate vector in the Hamiltonian, corresponding to the system dynamics.

$P_{b} \quad$ Battery power at its terminal.

$P_{b}^{*} \quad$ Optimal battery power at the terminal.

$P_{d} \quad$ Electric power demand at the output of the inverter.

$P_{f} \quad$ Fuel power.

$P_{b, \text { min }}$ Minimum battery power at the terminal.

$P_{c r} \quad$ A function of $\lambda$.

$P_{f, 0} \quad$ Engine fuel power at idling.

$P_{f, \max }$ Fuel power corresponding to $P_{g s}=P_{g s, \max }$, given by the genset's static map.

$P_{g s, \text { min }}$ Maximum electric power that the genset can use to brake.

$P_{g s} \quad$ Electric power of the genset.

$P_{p t r, \max }$ Maximum electric power that the powertrain can generate.

$P_{p t r \text { nom }}$ Nominal electric power that the powertrain can generate.

$Q \quad$ Available electric charge of the battery.

$Q_{\text {rated }}$ Rated capacity of the battery.

$Q_{l h v} \quad$ Lower heating value of the fuel. 
$R \quad$ Inner resistance of the battery.

$t_{s} \quad$ Sampling period.

$t_{c y c} \quad$ Total driving cycle time.

$T_{\text {em }} \quad$ Generator torque.

$T_{\text {ice }} \quad$ Engine torque.

$u \quad$ System input.

$U_{o c} \quad$ Open circuit voltage in the battery model.

$V_{b} \quad$ Terminal voltage of the battery.

$x \quad$ System state.

SOC State of charge.

\section{INTRODUCTION}

D UE to their ever-growing ubiquity, hybrid electric vehicles (HEV) have been a center of attention in automotive research with many endeavors to optimize their performance in order to meet customer satisfaction and minimize negative environmental impacts. One chief measure is fuel consumption which has direct effects on economy and environmental pollution and is of critical importance due to probable fossil fuel shortage. As a result, a considerable amount of research has been devoted to designing effective energy management systems (EMS) that aim at minimizing fuel consumption by finding optimal split of power between internal combustion engine (ICE) and on-board energy storage systems (ESS) [1] [3].

Dynamic programming (DP) is possibly the most studied framework for design and evaluation of EMSs [4], [5]. The methodology provides globally optimal solution to energy management problem by finding optimal trajectories through backward induction, but suffers from high computational complexity and the requirement that the entire drive cycles should be precisely known in advance. Due to its non-causal nature, DP cannot be implemented in real-time as it is impossible to know the exact future driving cycle conditions [6]. Hence, DP is usually regarded as an offline optimization method whose results can be used as benchmark to assess performance of other methods. Stochastic dynamic programming (SDP) has been employed to tackle the prior knowledge requirement of DP for real-time implementation by estimating future power demands using probabilistic framework [7], [8]. SDP achieves near-optimal results but has high computational burden that limits its usage in real-time [6], [9].

Another popular approach to energy management problem of HEVs is equivalent consumption minimization strategy (ECMS) which finds a suboptimal split of power by performing real-time minimization of an expression that consists of fuel and electric power terms. While numerous versions of ECMS exist in the literature [10], the optimal solution can be found by applying Pontryagin's minimum principle [11], [12]. ECMS is notably more time efficient than DP, but prior knowledge of drive cycle still remains a necessity.

Various adaptive versions of ECMS have been developed to tackle the cycle dependency issue by adaptively controlling the equivalence factor [13]-[15]. These methods go under the name Adaptive ECMS (A-ECMS), and can be generally put into three categories based on the employed adaptation principle: adaptation based on driving pattern recognition
[16]-[19], adaptation based on driving cycle prediction [20][22], and adaptation by instantaneous state of charge (SOC) feedback [13], [15], [23]-[26]. Methods in the first category use the observation that equivalence factors for statistically similar drive cycles are numerically close [10]. Equivalence factors for several drive cycles are precalculated and stored in a database. A window of past data is used and periodically updated to identify driving conditions using pattern recognition techniques. Equivalence factor is then regulated based on the identified conditions and the equivalence factor database. In the second category, future driving conditions are predicted, for example in terms of speed profiles or SOC trajectory, and equivalence factor is found by performing an optimization or through a direct formula based on the prediction. A common idea is to use PI controller to update the equivalence factor [22]. The last group of A-ECMS methods use instantaneously measured or estimated SOC to compute equivalence factor. Popular adaptation strategies are to use a PI controller [23], or just a simple proportional controller [15]. A more relaxed approach is to perform equivalence factor adaptation less frequently at fixed time intervals, instead of doing it at every instant, to encourage battery usage over wider range of SOC [13].

While majority of these methods offer promising results, their performances depend heavily on proper, usually adhoc, tuning of control parameters. Control design parameters might need to be recalibrated if a powertrain component is subjected to change, for example due to wear or replacement, or new drive cycles are introduced. In this light, a recently proposed adaptive ECMS technique uses estimates of equivalence factor bounds to establish a linear adaptation policy based on instantaneous SOC feedback [24]. Since there are no tunable parameters involved, tuning is not required for this method. A new version of the same methodology for parallel HEV is given in [25], which "softens" the linearity by introducing soft SOC bounds to avoid missing energy-saving opportunities. Of course, this comes with having tunable parameters.

In this paper, analytical solution to ECMS for series hybrid electric vehicles is derived. Similar problem has been addressed in [9], where two candidates for optimal solution are compared to each other and the one that corresponds to the smaller Hamiltonian expression is chosen. Unfortunately, admissibility of input signal is but partially taken into account while calculating optimal solutions, and as a result situations where none of the proposed candidates are optimal-or even feasible-are not explored. This problem will be addressed in the present work by dividing the set of positive sampled power demands into 4 disjoint subsets characterized by the equivalence factor. For each subset, admissible input set is determined and used to find the optimal solution. Additionally, a distinction is made between the maximum and nominal battery powers since the maximum achievable power is always smaller than the nominal value in practice. As will be shown in what follows, taking admissible input set into account requires more rigorous treatment as perceived previously. From the analytical solution, a bound will be established outside of which changing the equivalence factor does not bring about any change in the ECMS solution. Next, an adaptive ECMS 
approach will be proposed based on the analytical solution and the effective equivalence factor bounds. It should be noted that the analytical policy is applicable regardless of the adaptation mechanism that regulates the equivalence factor. Moreover, since the policy directly depends on model parameters, it automatically compensates for any change in those parameters. Effectiveness of the proposed analytical solution and the A-ECMS will be demonstrated for a hybrid electric wheel loader, and the repercussions of not incorporating drive cycle information into A-ECMS will be discussed.

The paper is organized as follows. Section II presents simplified models of relevant powertrain components and formulates the energy management problem in optimal control framework. Pontryagin's minimum principle is introduced and then used to solve the energy management problem. In section III, analytical optimal solution is derived and effective equivalence factor bounds are defined and presented. In section IV, the proposed analytical policy is verified for a hybrid electric wheel loader, where two drive cycles, namely short loading cycle and load and carry uphill, are considered. Based on the results from section III, an A-ECMS method is then proposed for series hybrid electric vehicles, and its performance is evaluated for 12 different drive cycles. Section V concludes the paper.

\section{PROBLEM FORMULATION}

\section{A. System description}

A simple illustration of the series hybrid powertrain is depicted in Fig. 1. At any moment, the driver demands/provides power by either pushing the gas pedal (positive power demand) or engaging the breaks (negative power demand), which is then translated into electric power $P_{d}$ at the output of the inverter and has to be supplied/utilized by the combination of the genset and the battery. The vehicle can operate in full electric drive, hybrid drive, battery recharging and regenerative braking modes. The set of electric power requests in a drive cycle, $\mathcal{P}_{d}$, can then be represented by a sequence of samples $P_{d}[k], k=0,1, \ldots, N_{c y c}$, derived by sampling power request $P_{d}(\cdot)$ every $t_{s}$ seconds for $t \in\left[0, t_{c y c}\right]$, i.e., $P_{d}[k]=P_{d}\left(k t_{s}\right)$ and $t_{c y c}=N_{c y c} t_{s}$. The objective of the EMS, as far as fuel economy is concerned, is to minimize fuel consumption for a driving mission by finding the optimal split of requested electric power between the energy sources, namely fossil fuel and electrochemical battery cells-losses in the inverter are ignored for simplicity.

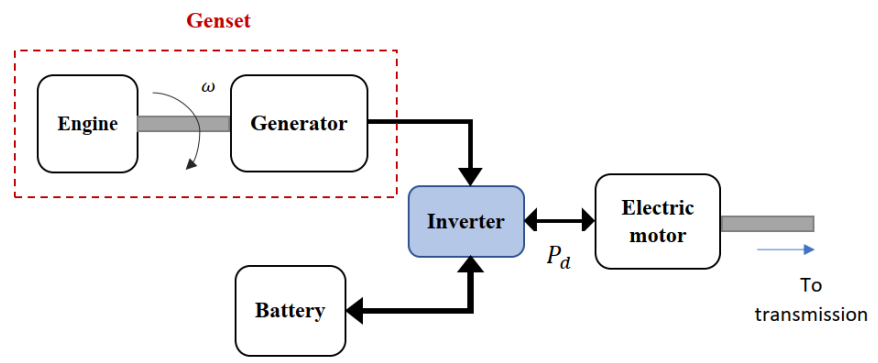

Fig. 1: Series hybrid powertrain.
1) Genset model: A Genset (or generator set) is the serial connection of internal combustion engine and generator, as illustrated in Fig. 1. The engine is modeled by a map that computes fuel power as a function of engine torque $T_{i c e}$ and shaft speed $\omega$. Similarly, the generator's electric power is given as a function of generator torque $T_{e m}$ and $\omega$. Denoting the shaft inertia by $J_{e}$, the dynamics relating the engine to the generator is simply written as

$$
T_{i c e}+T_{e m}=J_{e} \dot{\omega}
$$

In (1), the effect of parasitic torque is neglected. If the sampling time $t_{s}$ is large enough, this dynamics can be dismissed, and instead the genset model can be viewed as a static map that takes electric power as input and outputs the corresponding optimal fuel power. This map is generated by finding the pair of engine torque and speed that result in the minimal fuel consumption for any feasible electric power request of the genset. The map can be approximated by

$$
P_{f}= \begin{cases}P_{f, 0}+\gamma P_{g s}, & \text { if } P_{g s}>0, \\ 0, & \text { otherwise }\end{cases}
$$

where $P_{g s}$ is the genset's output power, $P_{f}$ is the fuel power, $P_{f, 0}$ is the fuel power during idling, and $\gamma$ is a dimensionless constant. The power $P_{g s, \max }$ designates the maximum electric power that the genset can produce, $P_{f, \max }$ denotes the fuel power corresponding to $P_{g s, \max }$ given by the static map, and $P_{g s, \text { min }}$ is the maximum possible braking power of the genset. The static map and the approximation (2) are depicted in Fig. 2 .

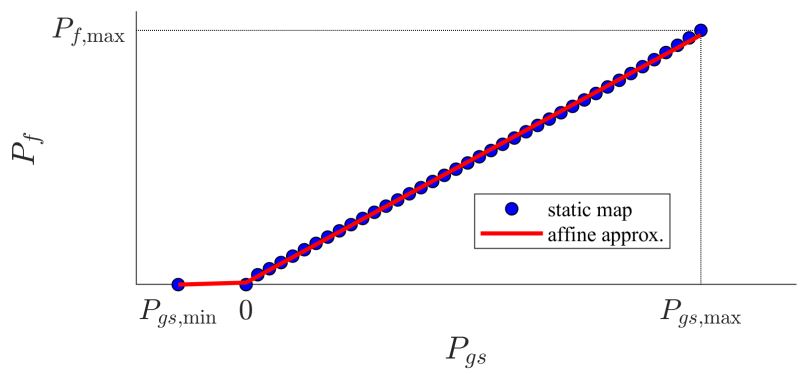

Fig. 2: Genset's static map (in blue) and the approximate model given by (2) (in red).

2) Battery model: The steady-state behavior of the battery is represented by an equivalent circuit model with the open circuit voltage $U_{o c}$ and the inner resistance $R$, as shown in Fig. 3. The current $I_{b}$ is calculated by

$$
I_{b}=\frac{U_{o c}-\sqrt{U_{o c}^{2}-4 R P_{b}}}{2 R},
$$

and the terminal power $P_{b}$ is given by $P_{b}=V_{b} I_{b}$. The SOC is defined as the ratio of the available electric charge $Q$ to the rated capacity $Q_{\text {rated }}$ as

$$
\mathrm{SOC}=\frac{Q}{Q_{\text {rated }}} .
$$




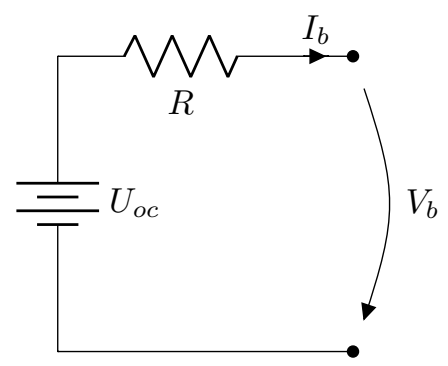

Fig. 3: Equivalent circuit model of the battery.

Assuming perfect charge/discharge efficiency, the battery dynamics is given by

$$
\text { SÖC }=-\frac{I_{b}}{Q_{\text {rated }}} .
$$

In (3) and (5), positive current (or power) corresponds to discharge mode and vice versa. Maximum and minimum terminal powers that the battery can provide and utilize are $P_{b, \text { max }}$ and $P_{b, \text { min }}$, respectively. In practice, $P_{b, \text { max }}$ is smaller than the nominal power $P_{b, \text { nom }}$, which is given by

$$
P_{b, \text { nom }}=\frac{U_{o c}^{2}}{4 R} .
$$

In this work, the maximum and the nominal powers that the powertrain can provide are respectively denoted by $P_{p t r \text {,max }}$ and $P_{p t r, \text { nom }}$, and they are defined by

$$
\begin{aligned}
& P_{p t r, \text { max }}:=P_{g s, \text { max }}+P_{b, \text { max }}, \\
& P_{p t r, \text { nom }}:=P_{g s, \text { max }}+P_{b, \text { nom }} .
\end{aligned}
$$

Clearly, a drive cycle characterized by the power sequence $\mathcal{P}_{d}$ is plausible only if $P_{d}[k] \leq P_{p t r, \max }, k=0,1, \ldots, N_{c y c}$.

\section{B. Energy management problem formulation}

Minimization of fuel consumption during a trip is mathematically equivalent to minimization of the index

$$
J_{f}=\int_{t_{i}}^{t_{f}} \dot{m}_{f} \mathrm{~d} t
$$

where $m_{f}$ denotes the mass of fuel consumed. The optimal solution must maintain energy balance at the power bus and must satisfy the input and state constraints. Taking $P_{b}$ as the input of the energy management controller, the optimal genset power is determined by $P_{d}(\cdot)-P_{b}(\cdot)$. Let the set of admissible input $\left(P_{b}\right)$ be denoted by $\mathcal{U}_{t}$, and the set of admissible state $(\mathrm{SOC})$ be denoted by $\mathcal{X} . \mathcal{U}_{t}$ is time dependent and is determined by considering the minimum and the maximum output power limits of the battery and the genset at any given time. In order to prevent acceleration of processes that degrade the battery, state of charge is prohibited to take extreme values that are close to 0 or 1 , and it is conventionally confined to $\mathcal{X}=[0.2,0.8]$. Charge sustaining policy mandates that the initial SOC should be recovered by the end of the driving mission.
The optimal solution is thus found by minimizing (9) while satisfying the constraints

$$
\begin{aligned}
& \operatorname{SOC}(t) \in \mathcal{X}, \\
& P_{b}(t) \in \mathcal{U}_{t}, \\
& \operatorname{SOC}\left(t_{f}\right)=\operatorname{SOC}(0),
\end{aligned}
$$

for all $t \in\left[0, t_{f}\right]$.

1) Pontryagin's minimum principle: The energy management problem described by the optimization index (9) and constraints (10) can be solved by Pontryagin's minimum principle. Consider the general problem of minimizing

$$
J_{P M P}=\varphi\left(x\left(t_{f}\right), t_{f}\right)+\int_{t_{i}}^{t_{f}} L(x(t), u(t), t) \mathrm{d} t,
$$

in which $x$ and $u$ are state and input vectors, respectively, and the system dynamics is given by

$$
\dot{x}=f(x(t), u(t), t) ; \quad x\left(t_{i}\right)=x_{0} .
$$

Assume that $x(t) \in \mathcal{X}$ and $u(t) \in \mathcal{U}$, for all $t \in\left[t_{i}, t_{f}\right]$, and that $\mathcal{X}$ can be written in the form $h(x(t), t) \leq 0$. Further assume that the minimizing solution to (11) must fulfill the final time constraint $\psi\left(x\left(t_{f}\right), t_{f}\right)=0$.

Suppose that $h(x(t), t) \leq 0$ is a $q$ th-order state variable inequality constraint, i.e., it should be differentiated at least $q$ times with respect to $t$ so that explicit dependence on $u(t)$ appears. Construct the Hamiltonian by adjoining the system dynamics and the $q$-time differentiated constraint $h^{(q)}(x(t), t)$ to the instantaneous cost $L(x(t), u(t), t)$ as

$$
\begin{aligned}
H= & H(x(t), u(t), p(t), t) \\
:= & L(x(t), u(t), t)+p(t)^{T} f(x(t), u(t), t) \\
& +\mu(t)^{T} h^{(q)}(x(t), t),
\end{aligned}
$$

where $p$ and $\mu$ are costate vectors. PMP states that the admissible optimal control must minimize the Hamiltonian alongside the optimal trajectory, i.e.,

$$
H\left(x^{*}(t), u^{*}(t), p^{*}(t), t\right) \leq H\left(x^{*}(t), u(t), p^{*}(t), t\right) .
$$

Furthermore, the dynamics for $p$ is given by

$$
\dot{p}^{*}(t)=-\frac{\partial H}{\partial x} .
$$

The final-time penalty $\varphi\left(x\left(t_{f}\right), t_{f}\right)$ and the constraint $\psi\left(x\left(t_{f}\right), t_{f}\right)=0$ yield the boundary condition for $p$ as

$$
p^{*}\left(t_{f}\right)=\left(\frac{\partial \varphi}{\partial x}+\frac{\partial \psi}{\partial x} \nu\right)_{t=t_{f}},
$$

where $\nu$ is such that $\psi\left(x\left(t_{f}\right), t_{f}\right)=0$ holds. If $h(x(t), t) \leq 0$ is inactive (i.e., $h(x(t), t) \neq 0), \mu(t)$ is zero, otherwise it is non-negative. On the constraint boundary, $h(x(t), t)=0$, and the costate $\mu(t)$ and the optimal control $u(t)$ are found by solving

$$
h^{(q)}(x(t), t)=0
$$

and the optimality condition

$$
\frac{\partial H}{\partial u}=0
$$


simultaneously [27]. The optimal trajectory is represented as the solution to the two-point boundary value problem given by (12), (15) and (16).

For the energy management problem, $\varphi\left(x\left(t_{f}\right), t_{f}\right)=0$, $L(x(t), u(t), t)=\dot{m}_{f}, f(x(t), u(t), t)$ is given by (5), $h(x(t))=[x(t)-0.8,0.2-x(t)]^{T}$, and $\psi\left(x\left(t_{f}\right), t_{f}\right)=$ $x\left(t_{f}\right)-x\left(t_{i}\right)$. SOC $\left(t_{i}\right)$ is typically considered to be 0.5 , and in practice SOC varies in a narrow interval around $\operatorname{SOC}\left(t_{i}\right)$ and does not reach the boundaries of the admissible set defined by $h(x(t)) \leq 0$. As a result, $h(x(t)) \leq 0$ usually does not become active. With this presumption, the Hamiltonian becomes

$$
\begin{aligned}
H & =\dot{m}_{f}+p \mathrm{SO} C \\
& =\frac{P_{f}}{Q_{l h v}}-p \frac{I}{Q_{\text {rated }}},
\end{aligned}
$$

where $Q_{l h v}$ is the lower heating value of the fuel. The voltage $U_{o c}$ is fairly constant if SOC variations are small, and thus derivative of SOC with respect to SOC can be considered zero. Hence, the costate dynamics becomes

$$
\dot{p}=0 \text {. }
$$

According to (16), the corresponding boundary condition is given by

$$
p\left(t_{f}\right)=\nu,
$$

where $\nu$ must ensure charge sustenance. Note that the optimal control formulation of energy management discussed above does not include explicit dependence on time. This implies that $t_{i}$ can be replaced by 0 for simplicity.

Now define $\lambda=\left(-Q_{l h v} / U_{o c} Q_{\text {rated }}\right) p$. Minimizing the Hamiltonian (19) is similar to minimizing

$$
H_{e c m s}=P_{f}+\lambda U_{o c} I,
$$

with $\dot{\lambda}=0 . \lambda$ is referred to as equivalence factor, and the terminology stems from the interpretation that $\lambda$ scales electrochemical power of the battery, $P_{e c h}=U_{o c} I$, and makes it comparable (or "equivalent") to fuel power in (22). Equation (22) represents the standard ECMS formulation. For the rest of the paper, $H_{\text {ecms }}$ is succinctly denoted by $H$.

\section{ANALYTICAL SOLUTiON}

For a given $\lambda$, the optimal solution can be found for any demand $P_{d}$ by minimizing (22). Depending on the drive mode, the Hamiltonian (22) can take two forms. For hybrid drive and battery-recharging modes, the Hamiltonian is given by

$$
H_{1}\left(P_{b}\right)=\gamma\left(P_{d}-P_{b}\right)+P_{f, 0}+\lambda U_{o c} \frac{U_{o c}-\sqrt{U_{o c}^{2}-4 R P_{b}}}{2 R},
$$

and for regenerative braking and electric drive modes, it will be

$$
H_{2}\left(P_{d}\right)=\lambda U_{o c} \frac{U_{o c}-\sqrt{U_{o c}^{2}-4 R P_{d}}}{2 R} .
$$

When both Hamiltonians are valid for a scenario, their minima (within the admissible set) must be compared to each other and the optimal control action corresponds to the Hamiltonian with the smaller value. Additionally, for cases where $H_{1}=$ $H_{2}, H_{2}$ (battery-only mode) is chosen to reduce emissions, noise and vibrations by switching off the engine [9]. The set of admissible inputs for $P_{d}(t)>0$ depends on $P_{d}$ and is determined by [28]

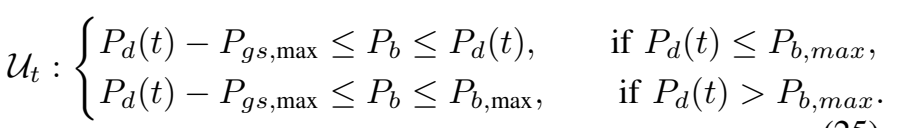

It is easy to show that the unconstrained minimum of (23) is achieved by

$$
\begin{aligned}
P_{b}^{*} & =P_{c r}(\lambda) \\
& :=\frac{U_{o c}^{2}}{4 R}\left(1-\frac{\lambda^{2}}{\gamma^{2}}\right),
\end{aligned}
$$

and the corresponding Hamiltonian is

$$
H_{1}\left(P_{c r}\right)=\gamma P_{d}+P_{f, 0}-\frac{U_{o c}^{2}}{4 R \gamma}(\gamma-\lambda)^{2} .
$$

In [9], the Hamiltonians (24) and (27) are compared to each other, but (25) is not taken into account to assess validity of the solution candidates.

Alternatively to (26), for a given $P_{c r}$, the corresponding equivalence factor $\lambda_{P_{c r}}$ can be computed by

$$
\begin{aligned}
\lambda_{P_{c r}} & =f_{\lambda}\left(P_{c r}\right) \\
& :=\gamma \sqrt{1-\frac{4 R P_{c r}}{U_{o c}^{2}}} .
\end{aligned}
$$

Note that since $P_{c r}$ is the only minima, $d H_{1} / d P_{b}$ is positive for $P_{b}>P_{c r}$ and it is negative for $P_{b}<P_{c r}$.

For a given $\lambda$, the set of positive sampled power demands $\stackrel{+}{\mathcal{P}}_{d}$ is the union of the disjoint sets defined by

$$
\begin{aligned}
& \stackrel{+}{\mathcal{P}}_{d, 1}:=\left\{P_{d} \in \stackrel{+}{\mathcal{P}}_{d} \mid P_{d}>P_{b, \max }\right\} \\
& \stackrel{+}{\mathcal{P}}_{d, 2}:=\left\{P_{d} \in \stackrel{+}{\mathcal{P}}_{d} \mid P_{c r}+P_{g s, \max }<P_{d} \leq P_{b, \max }\right\} \\
& \stackrel{+}{\mathcal{P}}_{d, 3}:=\left\{P_{d} \in \stackrel{+}{\mathcal{P}}_{d} \mid P_{c r}<P_{d} \leq \min \left(P_{c r}+P_{g s, \max }, P_{b, \text { max }}\right)\right\}
\end{aligned}
$$

and

$\stackrel{+}{\mathcal{P}}_{d, 4}:=\left\{P_{d} \in \stackrel{+}{\mathcal{P}}_{d} \mid P_{d} \leq \min \left(P_{c r}, P_{b, \max }\right)\right\}$.

Depending on $\lambda$, some of these sets might be empty.

Start-stop policy is such that the engine is switched on only if $P_{d}, P_{g s}>0$, otherwise it is shut off. Also, optimal solution to $P_{d}<0$ is clearly $P_{b}^{*}=P_{d}$, since the optimal strategy should recuperate as much power as possible during regenerative braking periods as battery wear is not of concern in this work [29]. In the sequel, the attention will be focused on $P_{d}>0$ and the optimal solution is found separately for cases (29-32).

\section{A. $P_{d} \in \stackrel{+}{\mathcal{P}}_{d, 1}$}

The battery-only mode is not possible for this case. Thus, the only valid Hamiltonian is $H_{1}$. According to (25), if $P_{d}>P_{g s, \max }+P_{c r}$, minimum of $H_{1}$ is given by $P_{d}-P_{g s, \max }$, otherwise $P_{c r}$ is the minima, provided that it does not exceed 
$P_{b, \max }$. Hence, for $P_{d} \leq P_{g s, \max }+P_{c r}$, if $\lambda<\lambda_{b}^{+}:=$ $f_{\lambda}\left(P_{b, \max }\right)$, minimum is achieved by $P_{b}^{*}=P_{b, \text { max }}$. The optimal solution for $\stackrel{+}{\mathcal{P}}_{d, 1}$ is thus

$$
P_{b}^{*}= \begin{cases}P_{b, \text { max }}, & \text { if } \lambda<\lambda_{b}^{+}, \\ P_{c r}, & \text { if } \lambda \geq \lambda_{b}^{+}, P_{d} \leq P_{c r}+P_{g s, \max }, \\ P_{d}-P_{g s, \max }, & \text { if } \lambda \geq \lambda_{b}^{+}, P_{d}>P_{c r}+P_{g s, \text { max }} .\end{cases}
$$

\section{B. $P_{d} \in \stackrel{+}{\mathcal{P}}_{d, 2}$}

Regardless of the values in $\stackrel{+}{\mathcal{P}}_{d}, \stackrel{+}{\mathcal{P}}_{d, 2}=\varnothing$ if $P_{c r} \geq P_{b, \text { max }}-$ $P_{g s, \text { max }}$ or equivalently if

$$
\lambda \leq f_{\lambda}\left(P_{b, \max }-P_{g s, \text { max }}\right)=\gamma \sqrt{1-\frac{4 R\left(P_{b, \max }-P_{g s, \max }\right)}{U_{o c}^{2}}} .
$$

Hence, assume $\lambda>f_{\lambda}\left(P_{b, \max }-P_{g s, \max }\right)$. Possible Hamiltonian candidates are $H_{1}\left(P_{d}-P_{g s, \max }\right)$ and $H_{2}\left(P_{d}\right)$. Note that $P_{c r}$ is not admissible for $\stackrel{+}{\mathcal{P}}_{d, 2}$. Let's investigate situations where $P_{d}-P_{g s, \max }$ is the optimal solution:

$$
\begin{gathered}
H_{1}\left(P_{d}-P_{g s, \text { max }}\right)<H_{2}\left(P_{d}\right) . \\
\Rightarrow \gamma P_{g s, \text { max }}+P_{f, 0}+\frac{\lambda U_{o c}}{2 R} \sqrt{U_{o c}^{2}-4 R P_{d}}< \\
\frac{\lambda U_{o c}}{2 R} \sqrt{U_{o c}^{2}-4 R\left(P_{d}-P_{g s, \text { max }}\right) .}
\end{gathered}
$$

Squaring both sides of (36) gives

$$
\begin{aligned}
\left(\gamma P_{g s, \max }+P_{f, 0}\right) \frac{\lambda U_{o c}}{R} \sqrt{U_{o c}^{2}-4 R P_{d}}< \\
\frac{\lambda^{2} U_{o c}^{2}}{R} P_{g s, \max }-\left(\gamma P_{g s, \max }+P_{f, 0}\right)^{2}
\end{aligned}
$$

For (37) to be feasible, it is necessary that its right-hand-side is positive, i.e.,

$$
\lambda>\frac{\gamma P_{g s, \max }+P_{f, 0}}{U_{o c}} \sqrt{\frac{R}{P_{g s, \max }}} .
$$

This is however guaranteed from the assumption $\lambda>$ $f_{\lambda}\left(P_{b, \max }-P_{g s, \max }\right)$ since

$$
\begin{aligned}
\lambda & >f_{\lambda}\left(P_{b, \text { max }}-P_{g s, \text { max }}\right) \\
& >f_{\lambda}\left(P_{b, \text { nom }}-P_{g s, \text { max }}\right) \\
& =\frac{2 \gamma P_{g s, \text { max }}}{U_{o c}} \sqrt{\frac{R}{P_{g s, \text { max }}}} \\
& \gg \frac{\gamma P_{g s, \text { max }}+P_{f, 0}}{U_{o c}} \sqrt{\frac{R}{P_{g s, \text { max }}}},
\end{aligned}
$$

where $P_{b, \text { max }}<P_{b, \text { nom }}$ and $\gamma P_{g s, \text { max }} \gg P_{f, 0}$ were used. From (37),

$$
\begin{aligned}
\sqrt{U_{o c}^{2}-4 R P_{d}}< & \frac{\lambda U_{o c}}{\gamma P_{g s, \max }+P_{f, 0}} P_{g s, \text { max }} \\
& -\frac{\gamma P_{g s, \max }+P_{f, 0}}{\lambda U_{o c}} R .
\end{aligned}
$$

For brevity of notation, define $\xi=\lambda U_{o c} /\left(\gamma P_{g s, \text { max }}+P_{f, 0}\right)$, and denote the right-hand-side of (40) by $\Gamma$. Then $\Gamma=\xi P_{g s, \max }-$ $R / \xi$, and (40) results in

$$
P_{d}>\frac{U_{o c}^{2}-\Gamma^{2}}{4 R} .
$$

This means that if $P_{d} \in \stackrel{+}{\mathcal{P}}_{d, 2}$ is larger than $P_{t h, 1}=$ $\left(U_{o c}^{2}-\Gamma^{2}\right) / 4 R$, the optimal solution is $P_{b}^{*}=P_{d}-P_{g s, \max }$, otherwise $P_{b}^{*}=P_{d}$. It can be shown that regardless of $\lambda$, for every demand in $\stackrel{+}{\mathcal{P}}_{d, 2}$ (41) is satisfied and that the optimal control is given by $P_{b}^{*}=P_{d}-P_{g s, \text { max }}$ iff

$$
P_{f, 0} \leq \gamma\left(\sqrt{P_{p t r, \text { nom }}}-\sqrt{P_{b, \text { nom }}}\right)^{2} .
$$

To see this, note that the curve $P_{t h, 1}$ lies below $P_{c r}+P_{g s, \max }$ if

$$
\frac{U_{o c}^{2}-\Gamma^{2}}{4 R}<P_{c r}+P_{g s, \max } .
$$

This leads to

$$
\frac{\left(\gamma P_{g s, \max }+P_{f, 0}\right)^{2}}{\gamma^{2}} \xi^{2}<\left(\xi P_{g s, \max }+\frac{R}{\xi}\right)^{2},
$$

from which

$$
\lambda<\frac{\gamma P_{g s, \max }+P_{f, 0}}{U_{o c}} \sqrt{\frac{R \gamma}{P_{f, 0}}} .
$$

Since $P_{d}>P_{c r}+P_{g s, \text { max }}$ for every $P_{d} \in \stackrel{+}{\mathcal{P}}_{d, 2}$ by definition (30), the condition (45) suggests that (41) is satisfied if $\lambda$ is smaller than the coordinate $\lambda_{i n t}$ at which the curves $P_{t h, 1}$ and $P_{c r}+P_{g s, \max }$ intersect:

$$
\lambda_{i n t}=\frac{\gamma P_{g s, \max }+P_{f, 0}}{U_{o c}} \sqrt{\frac{R \gamma}{P_{f, 0}}} .
$$

Let's consider the case $\lambda \geq \lambda_{\text {int }}$. First, see that the $\lambda$ for which $P_{t h, 1}=0$ is

$$
\lambda_{t h, 1}^{0}=\frac{\left(\gamma P_{g s, \max }+P_{f, 0}\right)\left(U_{o c}+\sqrt{U_{o c}^{2}+4 R P_{g s, \max }}\right)}{2 U_{o c} P_{g s, \max }},
$$

and the $\lambda$ for which $P_{c r}+P_{g s, \max }=0$ is given by

$$
\lambda_{g s}^{-}=f_{\lambda}\left(-P_{g s, \max }\right) \text {. }
$$

It is straight-forward to demonstrate that

$$
\lambda_{t h, 1}^{0} \leq \lambda_{g s}^{-} \Longleftrightarrow P_{f, 0} \leq \gamma\left(\sqrt{P_{p t r, \text { nom }}}-\sqrt{P_{b, \text { nom }}}\right)^{2} .
$$

For $\lambda>f_{\lambda}\left(P_{b, \text { max }}-P_{g s, \text { max }}\right)$, the curves $P_{c r}+P_{g s, \text { max }}$ and $P_{t h, 1}$ are strictly decreasing, and they only intersect at $\lambda_{\text {int }}$. Now since $P_{c r}+P_{g s, \max }$ lies above $P_{t h, 1}$ for $\lambda<\lambda_{i n t}$ according to (45), at the intersection,

$$
P_{t h, 1}\left(\lambda=\lambda_{\text {int }}\right) \leq 0 \Longleftrightarrow \lambda_{t h, 1}^{0} \leq \lambda_{g s}^{-} .
$$

It should be noted that $\lambda>f_{\lambda}\left(P_{b, \max }-P_{g s, \max }\right)$ is inferred by non-emptyness of $\stackrel{+}{\mathcal{P}}_{d, 2}$ from an earlier discussion. Considering that $P_{t h, 1}$ is strictly decreasing for $\lambda \geq \lambda_{\text {int }}$, a negative $P_{t h, 1}\left(\lambda=\lambda_{\text {int }}\right)$ implies that the condition (41) is 
automatically satisfied for any $\lambda \geq \lambda_{\text {int }}$ and $P_{d} \in \stackrel{+}{\mathcal{P}}_{d, 2}$, and vice versa. This together with (49) and (50) prove that for any positive power demand in $\stackrel{+}{\mathcal{P}}_{d, 2}$ and any $\lambda$, (41) is fulfilled iff (42) is valid. If (42) is not satisfied, at the intersection, $P_{t h, 1}\left(\lambda_{\text {int }}\right)>0$, and the optimal control can become $P_{b}^{*}=P_{d}$ as (41) is not guaranteed for $\lambda_{\text {int }} \leq \lambda \leq \lambda_{t h, 1}^{0}$. Graphical representation of the optimal solutions for $\stackrel{+}{\mathcal{P}}_{d, 1}$ and $\stackrel{\mathcal{P}}{d, 2}_{\text {are il- }}$ lustrated in Fig. 4 in different colors. For $\lambda>\max \left(\lambda_{g s}^{-}, \lambda_{t h, 1}^{0}\right)$, any $P_{d}>0$ belongs to $\stackrel{+}{\mathcal{P}}_{d, 1} \cup \stackrel{+}{\mathcal{P}}_{d, 2}$, and that $P_{b}^{*}=P_{d}-P_{g s, \text { max }}$. It is worth mentioning that the property (42) is an intrinsic characteristic of the powertrain and is independent of the drive cycle.

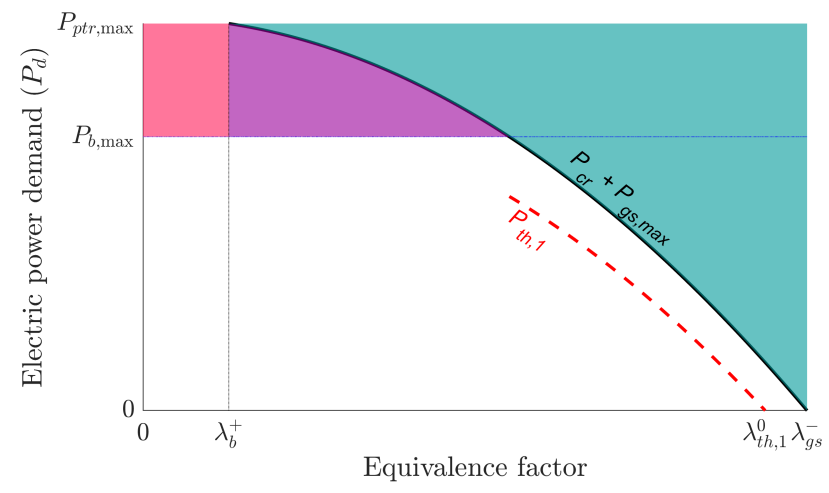

(a) $P_{f, 0} \leq \gamma\left(\sqrt{P_{p t r, \text { nom }}}-\sqrt{P_{b, \text { nom }}}\right)^{2}$.

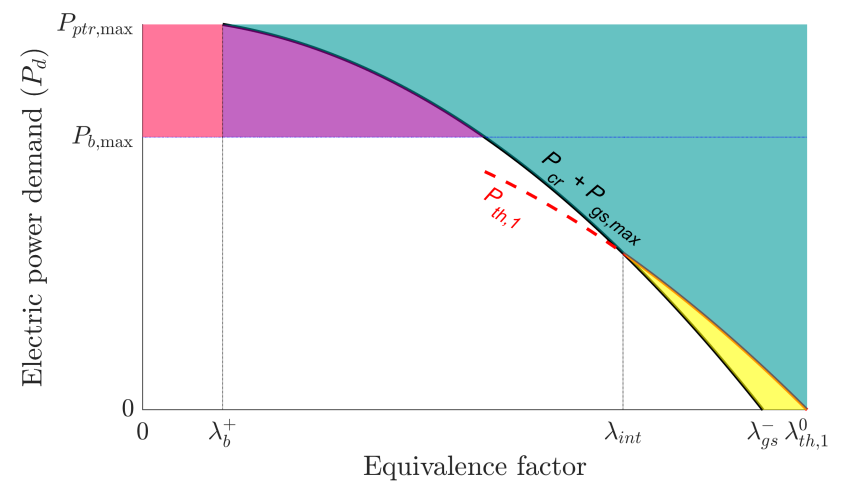

(b) $P_{f, 0}>\gamma\left(\sqrt{P_{p t r, \text { nom }}}-\sqrt{P_{b, \text { nom }}}\right)^{2}$.

Fig. 4: Analytical solution for $P_{d} \in \stackrel{+}{\mathcal{P}}_{d, 1} \cup \stackrel{+}{\mathcal{P}}_{d, 2}$, based on condition (42), illustrated in different colors: $\square\left(P_{b}^{*}=P_{c r}\right)$,

$\left(P_{b}^{*}=P_{d}-P_{g s, \max }\right), \quad\left(P_{b}^{*}=P_{d}\right), \square\left(P_{b}^{*}=P_{b, \max }\right)$.

Remark 1: Suppose that the nominal battery power is a times the maximum genset power, i.e., $P_{b, \text { nom }}=a P_{g s \text {, max }}$. Inequality (42) is equivalent to

$$
a \leq \frac{\left(1-\frac{P_{f, 0}}{\gamma P_{g s, \text { max }}}\right)^{2}}{4 \frac{P_{f, 0}}{\gamma P_{g s, \text { max }}}} .
$$

Since $P_{f, 0} \ll \gamma P_{g s, \max }$, the right-hand-side of (51) is considerably larger than 1 . As an example, for $P_{g s, \max }=80 \mathrm{~kW}$, $P_{f, 0}=1.8 \mathrm{~kW}$ and $\gamma=2.7$, (51) becomes

$$
a \leq 29.5 \text {, }
$$

which is well satisfied for most vehicles. Therefore, the situation in Fig. $4 \mathrm{~b}$ occurs if the powertrain consists of a very large battery system relative to the genset, where the vehicle is designed for mostly electric drive mode.

C. $P_{d} \in \stackrel{+}{\mathcal{P}}_{d, 3}$

The Hamiltonian candidates are $H_{1}\left(P_{c r}\right)$ and $H_{2}\left(P_{d}\right)$. Letting $H_{1}<H_{2}$ gives

$$
\gamma P_{d}+P_{f, 0}-\frac{U_{o c}^{2}}{4 R \gamma}(\gamma-\lambda)^{2}<\lambda U_{o c} \frac{U_{o c}-\sqrt{U_{o c}^{2}-4 R P_{d}}}{2 R} .
$$

It is straight-forward to show that (53) amounts to either

$$
\sqrt{U_{o c}^{2}-4 R P_{d}}>\frac{\lambda U_{o c}+2 \sqrt{R \gamma P_{f, 0}}}{\gamma},
$$

or

$$
\sqrt{U_{o c}^{2}-4 R P_{d}}<\frac{\lambda U_{o c}-2 \sqrt{R \gamma P_{f, 0}}}{\gamma} .
$$

From (31), $\sqrt{U_{o c}^{2}-4 R P_{d}} \leq \lambda U_{o c} / \gamma$, and thus (54) is not feasible. Inequality (55) necessitates

$$
\lambda>\frac{2 \sqrt{R \gamma P_{f, 0}}}{U_{o c}},
$$

and gives the following condition for $P_{d}$

$$
P_{d}>\frac{\left(\frac{-U_{o c}^{2}}{4 R}\right) \lambda^{2}+\left(\sqrt{\frac{\gamma P_{f, 0}}{R}} U_{o c}\right) \lambda-\gamma P_{f, 0}+\frac{\gamma^{2} U_{o c}^{2}}{4 R}}{\gamma}
$$

Denote the right-hand-side of (57) by $P_{t h, 2}$. Inequality (57) is possible only if $P_{t h, 2} \leq P_{b, \max }$ or equivalently,

$$
\begin{aligned}
\lambda & \geq \frac{2 \sqrt{R \gamma P_{f, 0}}}{U_{o c}}+\frac{2 R \gamma}{U_{o c}} \sqrt{\frac{P_{b, \text { nom }}-P_{b, \text { max }}}{R}} \\
& =\frac{2 \sqrt{R \gamma P_{f, 0}}}{U_{o c}}+\lambda_{b}^{+} .
\end{aligned}
$$

The right-hand-side of (58) is strictly greater than the righthand-side of (56). Consequently, the assumption (56) can be dropped as validity of (57) automatically implies (56). Consider that

$$
\begin{aligned}
P_{t h, 2} & =P_{c r}+\frac{1}{\gamma^{2}}\left(\sqrt{\frac{\gamma P_{f, 0}}{R}} U_{o c} \lambda-\gamma P_{f, 0}\right) \\
& >P_{c r}+\frac{1}{\gamma^{2}}\left(\sqrt{\frac{\gamma P_{f, 0}}{R}} U_{o c} \frac{2 \sqrt{R \gamma P_{f, 0}}}{U_{o c}}-\gamma P_{f, 0}\right) \\
& =P_{c r}+\frac{P_{f, 0}}{\gamma},
\end{aligned}
$$

where (56) was used in the process. Hence, $P_{t h, 2}$ is always above $P_{c r}$. The curve $P_{t h, 2}$ crosses zero at

$$
\lambda_{t h, 2}^{0}=\frac{2}{U_{o c}} \sqrt{\gamma R P_{f, 0}}-\gamma .
$$


Now assume that (42) is satisfied. Then the maximum value for $\lambda_{t h, 2}^{0}$ is achieved by $P_{f, 0}=\gamma\left(\sqrt{P_{p t r, \text { nom }}}-\sqrt{P_{b, \text { nom }}}\right)^{2}$ as

$$
\begin{aligned}
\max \left(\lambda_{t h, 2}^{0}\right) & =\frac{\gamma}{U_{o c}}\left(\sqrt{U_{o c}^{2}+4 R P_{g s, \max }}-\left(\frac{1}{\sqrt{R}}+1\right) U_{o c}\right) \\
& <\frac{\gamma}{U_{o c}} \sqrt{U_{o c}^{2}+4 R P_{g s, \max }} \\
& =\lambda_{g s}^{-}
\end{aligned}
$$

Also, it is seen that $P_{t h, 2}$ intersects with $P_{c r}+P_{g s, \max }$ at $\lambda_{\text {int }}$, defined by (46), and that $P_{c r}+P_{g s, \max }>P_{t h, 2}$ iff $\lambda<\lambda_{\text {int }}$. This observation, (59) and (61) together imply that $P_{t h, 2}$ lies between the curves $P_{c r}$ and $P_{c r}+P_{g s, \max }$ for $P_{t h, 2}>0$ iff (42) is satisfied, otherwise $P_{t h, 1}, P_{t h, 2}$ and $P_{c r}+P_{g s, \max }$ intersect at $\lambda_{\text {int }}<\max \left(\lambda_{g s}^{-}, \lambda_{t h, 1}^{0}\right)$. Graphical representation of the solution for $P_{d} \in \stackrel{+}{\mathcal{P}}_{d, 3}$ is presented in Fig. 5.

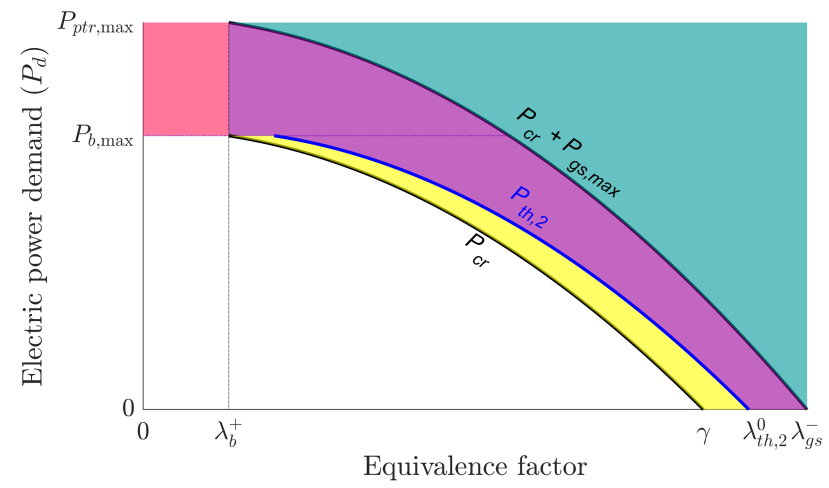

(a) $P_{f, 0} \leq \gamma\left(\sqrt{P_{p t r, \text { nom }}}-\sqrt{P_{b, \text { nom }}}\right)^{2}$.

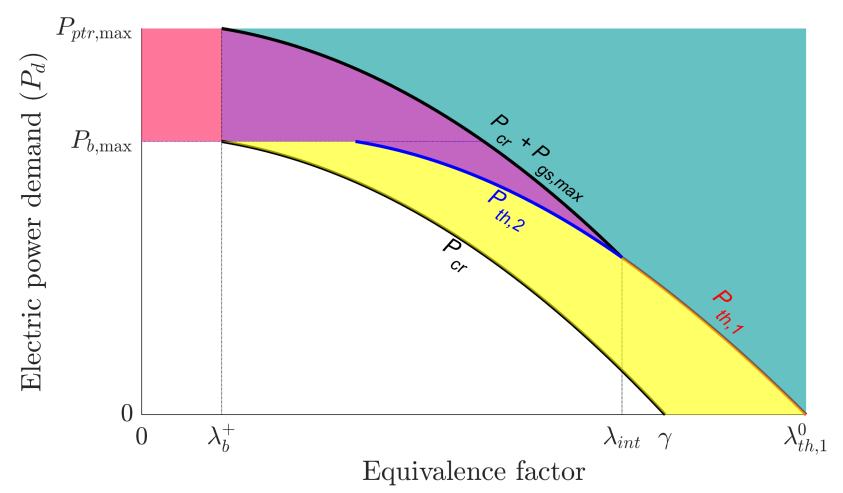

(b) $P_{f, 0}>\gamma\left(\sqrt{P_{p t r, \text { nom }}}-\sqrt{P_{b, \text { nom }}}\right)^{2}$.

Fig. 5: Analytical solution for $P_{d} \in \stackrel{\mathcal{P}}{d, 1}_{d} \cup \stackrel{+}{\mathcal{P}}_{d, 2} \cup \stackrel{+}{\mathcal{P}}_{d, 3}$, based on condition (42), illustrated in different colors: $\left(P_{b}^{*}=\right.$ $\left.P_{c r}\right), \square\left(P_{b}^{*}=P_{d}-P_{g s, \max }\right), \quad\left(P_{b}^{*}=P_{d}\right), \square\left(P_{b}^{*}=\right.$ $\left.P_{b, \text { max }}\right)$.

D. $P_{d} \in \stackrel{+}{\mathcal{P}}_{d, 4}$

For this case, minimum of $H_{1}$ is given by

$$
H_{1}\left(P_{d}\right)=P_{f, 0}+\lambda U_{o c} \frac{U_{o c}-\sqrt{U_{o c}^{2}-4 R P_{d}}}{2 R},
$$

which is clearly larger than

$$
H_{1}\left(P_{d}\right)=\lambda U_{o c} \frac{U_{o c}-\sqrt{U_{o c}^{2}-4 R P_{d}}}{2 R} .
$$

Therefore, the optimal control for $\stackrel{+}{\mathcal{P}}_{d, 4}$ is $P_{b}^{*}=P_{d}$. Analytical solution for all cases is depicted in Fig. 6. It should be noted that at the boundaries between the area for which $P_{b}^{*}=P_{d}$ (in yellow) and other areas, $P_{b}^{*}=P_{d}$ is favored for the reasons mentioned previously.

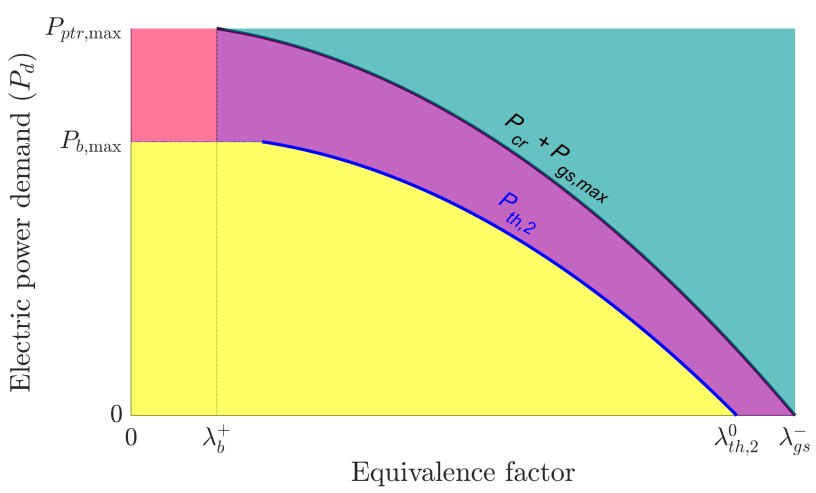

(a) $P_{f, 0} \leq \gamma\left(\sqrt{P_{p t r, \text { nom }}}-\sqrt{P_{b, \text { nom }}}\right)^{2}$.

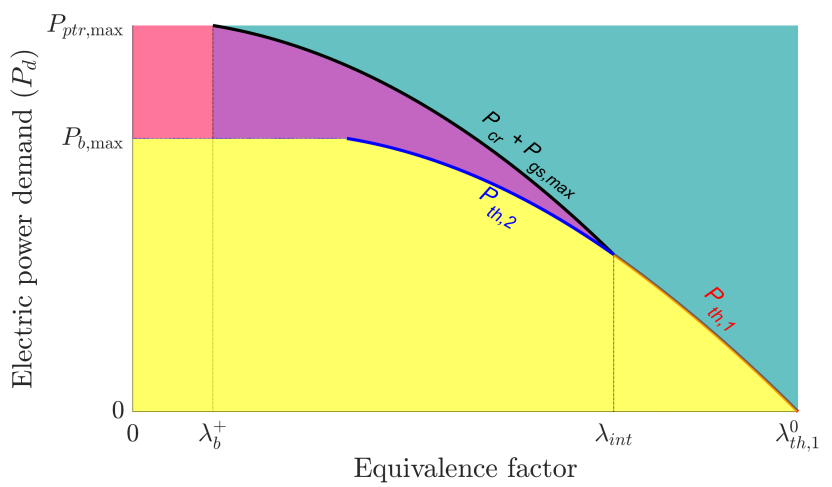

(b) $P_{f, 0}>\gamma\left(\sqrt{P_{p t r, \text { nom }}}-\sqrt{P_{b, \text { nom }}}\right)^{2}$.

Fig. 6: Analytical solution for all cases, based on condition (42), illustrated in different colors: $\square\left(P_{b}^{*}=P_{c r}\right), \square\left(P_{b}^{*}=\right.$ $\left.P_{d}-P_{g s, \max }\right), \quad\left(P_{b}^{*}=P_{d}\right), \square\left(P_{b}^{*}=P_{b, \max }\right)$.

\section{On equivalence factor bounds}

As it was shown previously, when $\lambda \geq \max \left(\lambda_{g s}^{-}, \lambda_{t h, 1}^{0}\right)$, the optimal solution for any $P_{d}>0$ is given by $P_{d}-P_{g s, \max }$, which is the lower bound of admissible set (25). Increasing $\lambda$ from this point on does not have any effect on the optimal solution. Thus, from (49) the maximum effective equivalence factor is calculated by

$$
\lambda_{\max }^{e f f}= \begin{cases}\lambda_{g s}^{-}, & P_{f, 0} \leq \gamma\left(\sqrt{P_{p t r, \text { nom }}}-\sqrt{P_{b, \text { nom }}}\right)^{2}, \\ \lambda_{t h, 1}^{0}, & P_{f, 0}>\gamma\left(\sqrt{P_{p t r, \text { nom }}}-\sqrt{P_{b, \text { nom }}}\right)^{2}\end{cases}
$$

From the charge sustenance identity

$$
\int_{0}^{t_{c y c}} I(t) \mathrm{d} t=0,
$$


$\lambda=\lambda_{\max }^{e f f}$ corresponds to a drive cycle for which

$$
\begin{gathered}
\int_{P_{d}(t)>0} \sqrt{U_{o c}^{2}-4 R\left(P_{d}(t)-P_{g s, \max }\right)} \mathrm{d} t \\
\quad+\int_{P_{d}(t) \leq 0} \sqrt{U_{o c}^{2}-4 R P_{d}(t)} \mathrm{d} t=t_{c y c} U_{o c} .
\end{gathered}
$$

Now suppose that $\lambda \leq \lambda_{b}^{+}$. This gives $P_{c r} \geq P_{b, \max }$ and $P_{c r}+P_{g s, \text { max }} \geq P_{p t r, \text { max }}$ which imply $\stackrel{+}{\mathcal{P}}_{d, 2}, \stackrel{+}{\mathcal{P}}_{d, 3}=\varnothing$. Consequently, $P_{d} \leq P_{c r}+P_{g s, \max }$ for any $P_{d} \in \mathcal{P}_{d}$ and the situations where $P_{b}^{*}=P_{d}-P_{g s, \max }$ never occur. The optimal battery power in this case is calculated by

$$
P_{b}^{*}= \begin{cases}P_{d}, & P_{d} \leq P_{b, \max } \\ P_{b, \max }, & P_{d}>P_{b, \max } .\end{cases}
$$

Hence, the minimum effective equivalence factor is

$$
\lambda_{\min }^{\text {eff }}=\lambda_{b}^{+},
$$

which is in practice non-zero since $P_{b, \max }<P_{b, \text { nom }}$.

The solution (67) corresponds to the full electric drive mode, except for $P_{d}>P_{b \text {,max }}$ where the genset also has to contribute minimally, and is possible given that there are enough periods of braking to replenish the battery, as there is no restriction on $\mathcal{P}_{d}$ in this respect. Such case can happen, for example, in a driving scenario that includes sufficient downhill driving phases so that regenerative braking can fully compensate for maximal effort of battery during traction. In particular, for a drive cycle with $P_{d} \leq P_{b \text {, max }}$, charge sustenance is fulfilled with $\lambda=\lambda_{b}^{+}$, i.e. equivalence factor is $\lambda_{b}^{+}$, if the curve $P_{d}(\cdot)$ satisfies

$$
\int_{0}^{t_{c y c}} \sqrt{U_{o c}^{2}-4 R P_{d}(t)} \mathrm{d} t=t_{c y c} U_{o c} .
$$

In the ideal case where $P_{b, \max }=P_{b, \text { nom }}, \lambda_{b}^{+}$is identically zero.

\section{Summary of the optimal policy}

For $P_{d}>0$, the optimal solution derived in the previous parts can be summarized as follows:

(i) $P_{f, 0} \leq \gamma\left(\sqrt{P_{p t r, \text { nom }}}-\sqrt{P_{b, \text { nom }}}\right)^{2}$ :

$$
\begin{aligned}
& \text { if } \lambda \leq \lambda_{b}^{+}, \\
& P_{b}^{*}= \begin{cases}P_{d}, & P_{d} \leq P_{b, \max }, \\
P_{b, \max }, & P_{d}>P_{b, \max },\end{cases}
\end{aligned}
$$

and if $\lambda>\lambda_{b}^{+}$,

$$
P_{b}^{*}=\left\{\begin{array}{l}
P_{d}, \quad P_{d} \leq P_{t h, 2}^{s a t}, \\
P_{c r}, \quad P_{t h, 2}^{s a t}<P_{d} \leq P_{c r}+P_{g s, \max }, \\
P_{d}-P_{g s, \max }, \quad P_{d}>P_{c r}+P_{g s, \max },
\end{array}\right.
$$

where $P_{t h, 2}^{s a t}$ denotes $\min \left(P_{b, \max }, P_{t h, 2}\right)$.

(ii) $P_{f, 0}>\gamma\left(\sqrt{P_{p t r, \text { nom }}}-\sqrt{P_{b, \text { nom }}}\right)^{2}$ : for $\lambda \leq \lambda_{b}^{+}$and $\lambda_{b}^{+}<\lambda \leq \lambda_{i n t}, P_{b}^{*}$ is given by (70) and (71), respectively. If $\lambda>\lambda_{\text {int }}$, the solution is

$$
P_{b}^{*}= \begin{cases}P_{d}-P_{g s, \max }, & P_{d}>P_{t h, 1}, \\ P_{d}, & P_{d} \leq P_{t h, 1} .\end{cases}
$$

Visual representations of these policies are shown in Fig. 6.

\section{CASE-STUdY}

\section{Verification of the analytical solution}

The analytical solution introduced earlier is assessed for a hybrid electric wheel loader. Simulations are performed for five drive cycles, but the results for only two of them, namely short loading cycle (SLC) and load and carry uphill (LCU), are presented. Fig. 7 illustrates vehicle's operation during SLC. The cycle begins at (4) where the wheel loader accelerates toward the pile and decelerates upon reaching 2 . After penetrating the pile at 1 and filling the bucket, the wheel loader is put into reverse and goes back to 4 . The operator puts the vehicle into forward and moves toward the load receiver located at 6 while lifting the bucket highly enough. After tilting the bucket and unloading material, it is put into reverse and gets back to the starting position while at the same time the bucket is lowered. In LCU, the distance from 4 to 6 is about 10 times larger than SLC [30].

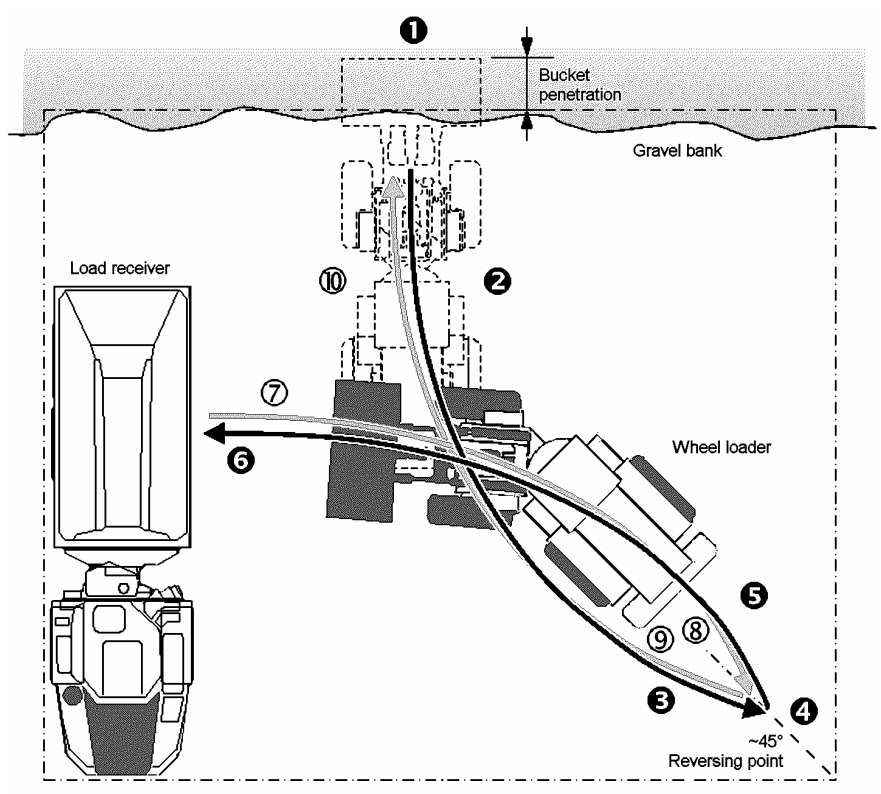

Fig. 7: Short loading cycle operation of wheel loader [31]

In the wheel loader under study, the engine and the generator's output powers are comparable in magnitude so that (42) is satisfied and the analytical solution is given by (70) and (71). In order to compare the solution derived by numerical minimization with the one from the proposed analytical solution, the value of equivalence factor is found by the Secant method [32]. To accomplish this, for two preset values of equivalence factor, $\lambda_{0}$ and $\lambda_{1}$, the respective final states of charge $\mathrm{SOC}_{0}\left(t_{c y c}\right)$ and $\mathrm{SOC}_{1}\left(t_{c y c}\right)$ are found by simulating optimal solutions through either minimizing the Hamiltonian 
numerically or the analytical solution provided previously, depending on the method under consideration. Optimal value of equivalence factor is found by the iteration

$$
\begin{aligned}
\lambda_{j}=\lambda_{j-1}-\left(\lambda_{j-1}-\lambda_{j-2}\right) & \\
\cdot & \frac{\operatorname{SOC}_{j-1}\left(t_{c y c}\right)-0.5}{\operatorname{SOC}_{j-1}\left(t_{c y c}\right)-\operatorname{SOC}_{j-2}\left(t_{c y c}\right)},
\end{aligned}
$$

for $j=2,3, \cdots, j_{\max }$ in which $\operatorname{SOC}_{j}\left(t_{c y c}\right)$ is the the final state of charge resulting from $\lambda=\lambda_{j}$. Iteration continues until either maximum number of iterations $j_{\max }$ is exceeded or $\operatorname{SOC}\left(t_{c y c}\right)$ is within the specified bounds around 0.5. In this work, the bounds are defined by relative error of \pm 0.01 . For the present application, equivalence factors are relatively close to $\gamma . \lambda_{0}=\gamma$ and $\lambda_{1}=\gamma+0.1$ were thus found to be appropriate choices for fast convergence of the Secant algorithm. Simulation results for the analytical and numerical methods are depicted in Fig. 8 and Fig. 9. It is evident that the state and control signal trajectories coincide for the two methods, which verifies the proposed analytical solution. Sim-

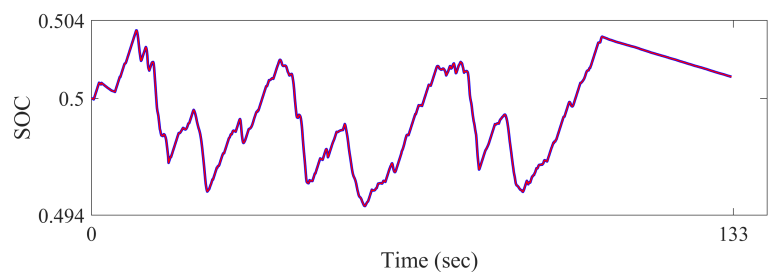

(a) Short loading cycle.

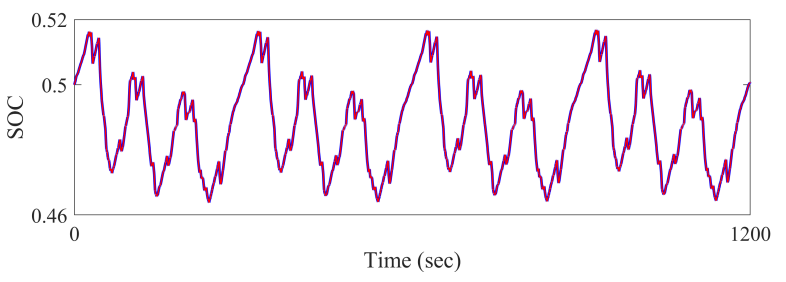

(b) Load and carry uphill.

Fig. 8: Comparison of SOC trajectories resulted from the analytical (red curves) and numerical (blue curves) methods.

ulation times for the methods, using the same computer, are provided in Table I. These values suggest that using analytical solution significantly decreases simulation time, making it a useful candidate for real-time implementation.

TABLE I: Simulation times for single drive cycle (in seconds)

\begin{tabular}{l|c|c}
\hline Drive cycle & Non-analytical method & Analytical method \\
\hline SLC & 6.61 & 0.15 \\
LCU & 45.73 & 0.97 \\
\hline
\end{tabular}

\section{Adaptive ECMS based on the analytical optimal solution}

The energy manager in the wheel loader consists of two modules, namely the cycle identifier that detects the ongoing drive cycle, and the controller which uses the information from the cycle identifier and equivalence factor database to generate the appropriate control signal. The equivalence factor

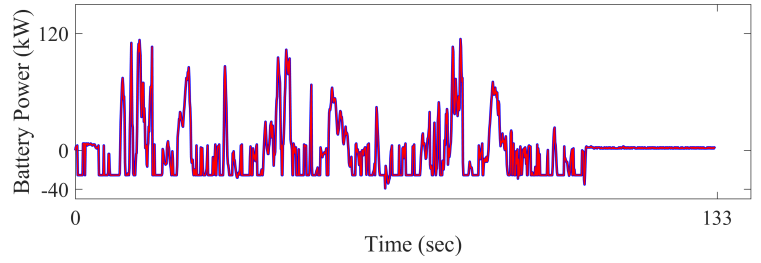

(a) Short loading cycle

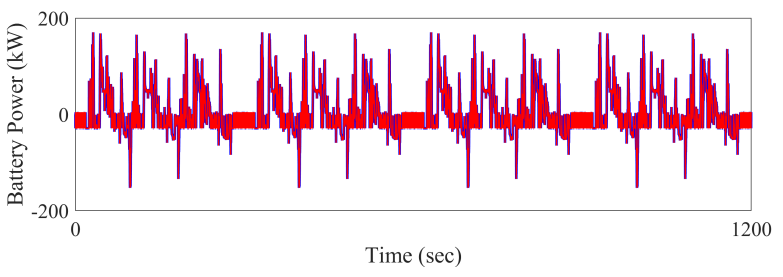

(b) Load and carry uphill

Fig. 9: Comparison of $P_{b}$ trajectories resulted from the analytical (red curves) and numerical (blue curves) methods.

$\lambda$ can be found by, for example, an iterative algorithm such as shooting method [10], given that power demand is precisely known beforehand. Since finding a $\lambda$ that fulfills the charge sustenance condition $\operatorname{SOC}\left(t_{c y c}\right)=\operatorname{SOC}(0)$ might not be possible, final state of charge is allowed to deviate from target value by a small predefined tolerance. If the same drive cycle is repeated consecutively, using the same $\lambda$ will cause the deviation to accumulate and become large over time, leading to violation of state of charge constraints and/or unfulfillment of charge sustenance policy at the least.

To tackle this problem, one remedy is to update $\lambda$ at the beginning of each drive cycle according to a precomputed equivalence factor lookup table that gives equivalence factor as a function of initial state of charge. This approach could work if the drive cycle is precisely known ahead of time so that the optimization is done in an offline fashion. Nevertheless, the non-causal nature of this assumption dissuades its practicality; even for vehicles that are expected to follow specific sets of drive cycles, drivers do not follow the nominal speed/power profiles exactly. In this case, using equivalence factors that correspond to nominal profiles for non-ideal drive cycles might lead to control signals that are far from optimal, beside violation of charge sustenance requirement. Additionally, this strategy does not incorporate feedback control but only after completion of a full drive cycle where final SOC is used to determine new equivalence factor for the upcoming drive cycle. If cycle detection algorithm fails to predict the ongoing drive cycle correctly for some reason, the EMS would not be able to correct equivalence factor before reaching the end of ongoing drive cycle, resulting in stability and optimality issues.

For a series HEV, suppose that the estimates of equivalence factors for possible drive cycles are known. For a vehicle whose operation is already known, this corresponds to equivalence factors calculated for nominal drive cycles. Consider 
the $\lambda$-adaptation strategy

$\lambda(t)=\left\{\begin{array}{l}\lambda_{\max }^{e f f}, \\ \lambda_{c y c}^{\mathrm{nom}}+r_{1}(\mathrm{SOC}-0.5), \\ \lambda_{c y c}^{\mathrm{nom}}-r_{2}(\mathrm{SOC}-0.5), \\ \lambda_{\min }^{e f f},\end{array}\right.$

$$
\begin{aligned}
0 & \leq \operatorname{SOC}(t)<\operatorname{SOC}_{L}, \\
\operatorname{SOC}_{L} & \leq \operatorname{SOC}(t)<0.5, \\
0.5 & \leq \operatorname{SOC}(t)<\operatorname{SOC}_{H}, \\
\operatorname{SOC}_{H} & \leq \operatorname{SOC}(t) \leq 1,
\end{aligned}
$$

where $\lambda_{c y c}^{\text {nom }}$ denotes the equivalence factor corresponding to the nominal drive cycle, and

$$
\begin{aligned}
& r_{1}=\frac{\lambda_{c y c}^{\mathrm{nom}}-\lambda_{\max }^{e f f}}{0.5-\mathrm{SOC}_{L}}, \\
& r_{2}=\frac{\lambda_{c y c}^{\mathrm{nom}}-\lambda_{\min }^{e f f}}{\mathrm{SOC}_{H}-0.5} .
\end{aligned}
$$

The values $\mathrm{SOC}_{L}$ and $\mathrm{SOC}_{H}$ respectively denote the minimum and maximum bounds that define $\mathcal{X}$ in (10). Graphical representation of (74) is demonstrated in Fig. 10. The strategy uses the minimum and maximum effective equivalence factors derived previously and incorporates nominal equivalence factor corresponding to the ongoing drive cycle. This requires the AECMS strategy to be implemented in conjunction with a drive cycle detection algorithm and an equivalence factor lookup table that includes equivalence factors for nominal scenarios.

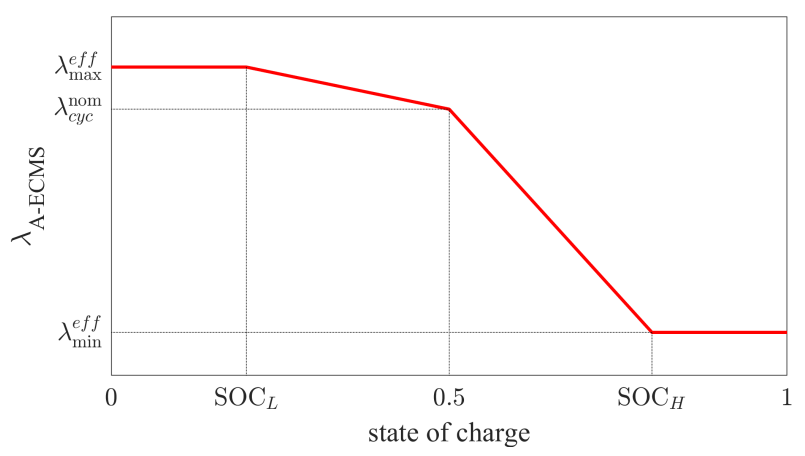

Fig. 10: Adaptive ECMS based on minimum and maximum effective equivalence factors and drive cycle information.

In what follows, the performance of the proposed A-ECMS (74) for series HEVs will be explored. The proposed strategy is implemented together with the analytical optimal solution derived in the previous section, and the goal is to compare its performance to the PMP-based solution computed numerically.

To simulate realistic driving scenarios due to imperfect driver model, for each drive cycle six non-ideal profiles are created based on ideal power demand data. Disturbance is induced by adding to the ideal power demand $\mathcal{P}_{d}$ a stochastic process that is characterized by power demand amplitude at any given sample. More specifically, the profiles are generated according to

$$
P_{d, i}^{\delta}[k]=P_{d}[k]\left(1+\delta_{i}(k)\right)
$$

at the sample points, where $\mathcal{P}_{d, i}^{\delta}:=\left\{P_{d, i}^{\delta}[k]\right\}_{k=1}^{N_{c y c}}$ is assumed to represent the non-ideal power demand and

$$
\delta_{i}(k) \sim \mathcal{U}_{k}\left(\underline{l}_{i}, \bar{l}_{i}\right)
$$

is a uniform random variable. $\left(\underline{l}_{i}, \bar{l}_{i}\right)=(-0.2,0.2)$ for $i=1,2,\left(\underline{l}_{i}, \bar{l}_{i}\right)=(-0.2,0.3)$ for $i=3,4$, and $\left(\underline{l}_{i}, \bar{l}_{i}\right)=$ $(-0.3,0.2)$ for $i=5,6$.

Simulations for one drive cycle demonstrate that the AECMS strategy limits battery usage to a narrower SOC band compared to the PMP solution for $\mathcal{P}_{d, i}^{\delta}, i=1,2,5,6$, see Fig. 11. This is mainly due to instantaneous adaptationor correction-of equivalence factor as SOC deviates from 0.5 . An opposite effect is observed for $\mathcal{P}_{d, 3}^{\delta}$ and $\mathcal{P}_{d, 4}^{\delta}$ where SOC operation range is increased. The main reason for this occurrence is that $\mathcal{P}_{d, 3}^{\delta}$ and $\mathcal{P}_{d, 4}^{\delta}$ tend to be more "aggressive" than nominal profiles (both in braking and traction) and their equivalence factors are slightly larger than $\lambda_{c y c}^{\text {nom }}$. As a result, the adaptive strategy forces the battery to discharge more than the PMP-based controller would, by which it decreases SOC range's lower limit. For all profiles, however, the differences between SOC ranges are infinitesimal.

For better assessment and comparison of the methods, it deems necessary to perform simulations for longer durations. Hence, simulations are done for one full day of operation, represented by 8 -hour repetition of each drive cycle. Fuel

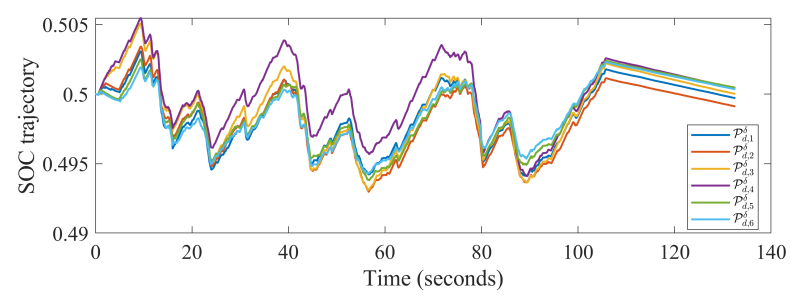

(a) ECMS

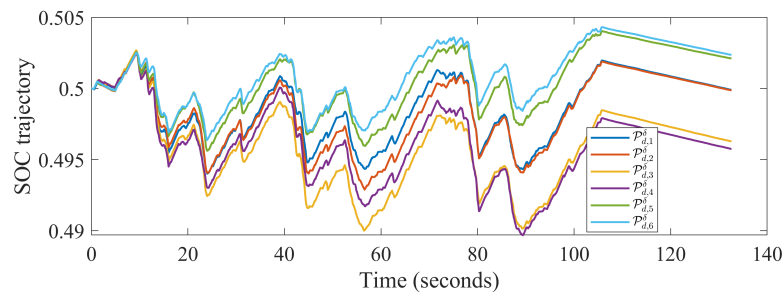

(b) Adaptive ECMS

Fig. 11: Comparison of SOC trajectories from ECMS and proposed A-ECMS for non-ideal SLC profiles.

consumption values for one day of operation, normalized with respect to the PMP results, are provided in Table II, and SOC values at the end of each cycle are depicted in Fig. 12 and Fig. 13, respectively corresponding to the proposed A-ECMS and PMP-based methods. It can be seen that the fuel consumption values are very close to those achieved through PMP-based optimal control. In some cases, A-ECMS leads to lower fuel consumption than the PMP does. This is chiefly due to smaller SOC levels at the end of simulation as comparison between Fig. 12 and Fig. 13 suggests. Besides, there are improvements in fuel economy that are attributed to accuracy of analytical solution whereas precision of numerical minimization depends on input discretization. In the simulation, a very fine grid was considered for $P_{b}$ where further increment in grid size would significantly increase simulation time. It is important to note that unlike the proposed A-ECMS, PMP results-by which 
the values in Table II are normalized-are not achievable in practice due to non-causality.

TABLE II: Normalized fuel consumption for one day of operation (in \%)

\begin{tabular}{|c|c|c|}
\hline Profile Drive cycle & SLC & $\mathrm{LCU}$ \\
\hline $\mathcal{P}_{d, 1}^{\delta}$ & 100.0022 & 100.0129 \\
\hline $\mathcal{P}_{d, 2}^{\delta}$ & 99.9940 & 100.0116 \\
\hline $\mathcal{P}_{d, 3}^{\delta}$ & 99.9829 & 100.0042 \\
\hline $\mathcal{P}_{d, 4}^{\delta}$ & 99.9813 & 99.9982 \\
\hline $\mathcal{P}_{d, 5}^{\delta}$ & 100.0009 & 100.0116 \\
\hline $\mathcal{P}_{d, 6}^{\delta}$ & 100.0088 & 100.0104 \\
\hline
\end{tabular}

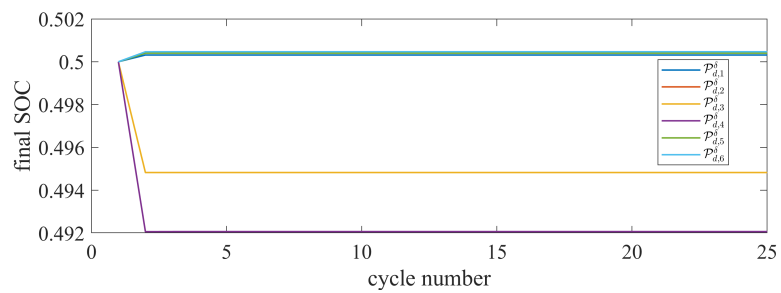

(a) LCU

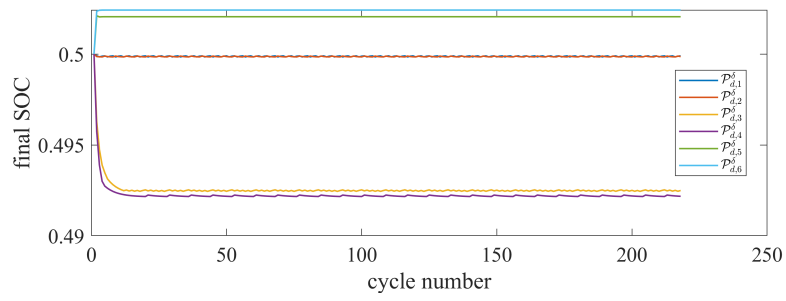

(b) SLC

Fig. 12: SOC values at the end of each drive cycle for nonideal profiles, corresponding to the proposed A-ECMS (74).

Simulation times for the proposed A-ECMS are significantly smaller than the PMP and while this is remarkable, it can be further improved by adapting $\lambda$ at fixed time periods while keeping it constant in between, instead of doing it at every instant. Another advantage of the proposed strategy is that it only requires a database for nominal/approximate equivalence factors for possible drive cycles. Implementation of ECMS in the way formerly described necessitates a much larger database that includes lookup tables for several profiles per drive cycle and each lookup table should consist of equivalence factors for different initial SOC values.

The importance of including drive cycle's information in AECMS strategy can be illustrated by comparing SOC trajectories resulted from the proposed method with the methodology presented in [24], in which estimates of equivalence factor bounds were used to construct a linear adaptation rule based on instantaneous SOC. The corresponding adaptation rule is depicted in Fig. 14. The main advantage of this method is that it does not require parameter tuning.

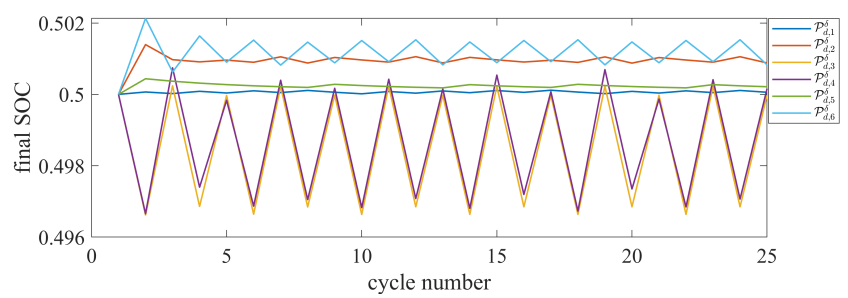

(a) LCU

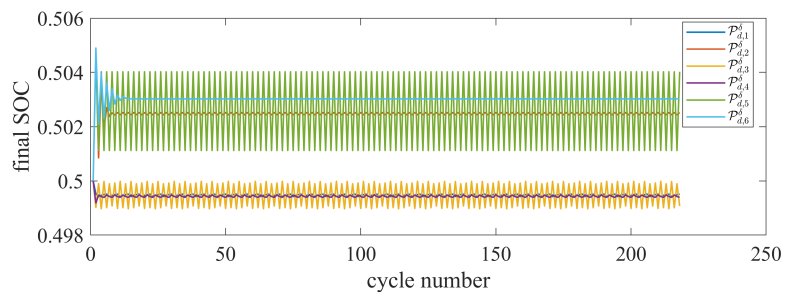

(b) SLC

Fig. 13: SOC values at the end of each drive cycle for nonideal profiles, corresponding to ECMS solution.

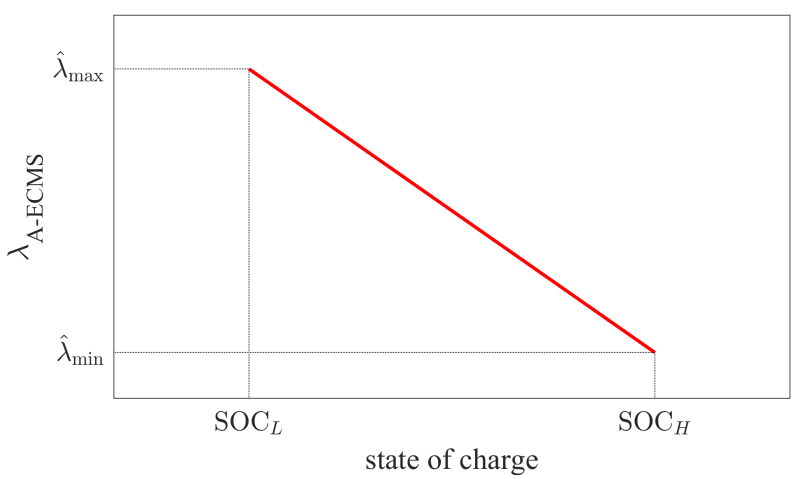

Fig. 14: Proposed adaptive ECMS based on estimate min/max equivalence factors [24].

SOC values after each drive cycle are depicted in Fig. 15. It can be seen that SOC values at the end of each cycle are far from 0.5 , and are closer to the lower bound $\mathrm{SOC}_{L}=0.2$. The reason behind this is that when $\mathrm{SOC}$ reaches 0.5 , adaptive algorithm passes average of equivalence factor bounds as instantaneous $\lambda$ to the optimizer but this value is considerably smaller than optimal equivalence factors. This dissimilarity leads battery system to over-discharge and the vehicle has to start subsequent cycles with depleted battery. To address this issue, one idea is to incorporate approximations of equivalence factors for different scenarios. The effect of such action has been already seen in Fig. 12 where nominal equivalence factors were used.

Remark 2: For the Energy management problem at hand, $h(x(t))=[x(t)-0.8,0.2-x(t)]^{T} \leq 0$ is a first order state inequality constraint. Thus, the Hamiltonian (13) becomes

$$
\begin{aligned}
H & =\dot{m}_{f}+\left(p+\mu_{1}-\mu_{2}\right) \mathrm{SO} \mathrm{C} \\
& =\dot{m}_{f}+p^{c} \mathrm{SOOC},
\end{aligned}
$$

in which $\mu=\left[\mu_{1}, \mu_{2}\right]^{T}$ and $p^{c}:=p+\mu_{1}-\mu_{2}$. At the boundary of the state constraint, either $\mu_{1}$ or $\mu_{2}$ is positive 


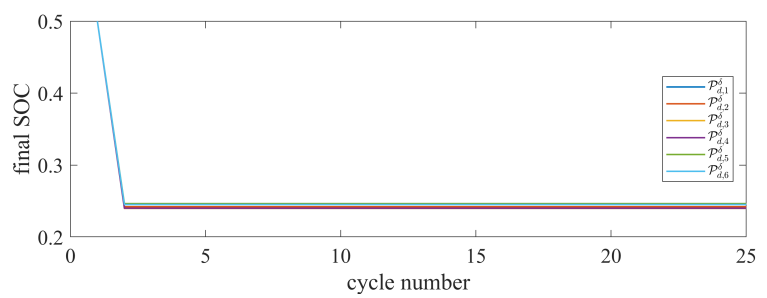

(a) LCU

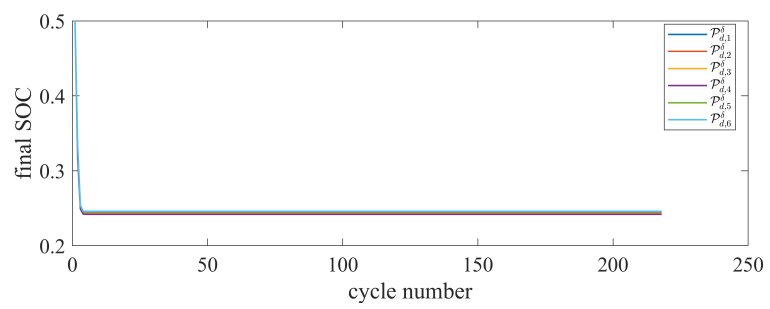

(b) SLC

Fig. 15: SOC values at the end of each drive cycle for nonideal profiles, corresponding to the A-ECMS method in [24].

and the other one is zero. For example, if $\operatorname{SOC}(t)=0.8$ for some $t, \mu_{1}>0$ and $\mu_{2}=0$ which gives $p^{c}>p$. This leads to a control signal that encourages more battery usage to reduce SOC. In this case, strongest control action to keep SOC within the bounds or push it into interior of $\mathcal{X}$ is achieved by increasing $p^{c}$ to the point where no change in control signal is observed. This corresponds to reducing equivalence factor to the point where the vehicle works in battery-only mode (except for $P_{d}>P_{b, \max }$ ), which was shown previously to be the minimum effective equivalence factor computed by (68). Similarly, if $\operatorname{SOC}(t)=0.2, p^{c}<p$ and largest control action that guarantees $\mathrm{SOC} \geq 0.2$ is commanded when equivalence factor attains its maximum effective value. This idea is captured in (74). It is worth noting that unlike standard ECMS where $p^{c}$ is constant when not on the boundary, the proposed A-ECMS changes equivalence factor (and hence the costate) dynamically to compensate for SOC moving away from target SOC $(=0.5)$. Even in cases where equivalence factor is not the same as $\lambda_{c y c}^{\text {nom }}$, which is expected in practice, when SOC gets close to state constraints bounds, equivalence factor is very close to either $\lambda_{\max }^{e f f}$ or $\lambda_{\min }^{e f f}$ so that the resulting control is already vigorously pushing SOC toward the target value. This will guarantee satisfaction of SOC constraints and the price to pay is a small increase in fuel consumption which was shown to be negligible.

\section{CONCLUSION}

Analytical optimal solution for energy management of series hybrid electric vehicles was addressed in this paper. The problem was formulated in an optimal control framework and was solved by Pontryagin's minimum principle. Since the admissible input set depends on power demand at any given time, the set of positive electric power demands was divided into four disjoint subsets, and for each case the optimal control was found separately. In the process, nominal battery power and maximum battery power were considered different as it is the case in reality. It was seen that the optimal input candidates depend on the power demand and the equivalence factor, and that there is a total of four candidates for the optimal battery power. Additionally, the analytical policy changes slightly depending on a condition that is characterized solely on the powertrain's characteristics. Since the analytical policy was derived without restricting or controlling the equivalence factor, it is applicable regardless of the mechanism that governs the equivalence factor. From the derived solution, minimum and maximum effective equivalence factors were found. It was shown that reducing/increasing equivalence factor respectively below or beyond these values does not affect optimal controls regardless of drive cycle. Two drive cycles, namely short loading cycle and load and carry uphill, were used to verify the analytical solution for a series hybrid wheel loader. Using the effective bounds, an adaptive energy management strategy was proposed. The strategy combines the previously found analytical policy with a simple adaptation scheme for equivalence factor. Performance of the proposed A-ECMS was examined by performing one-day simulations of 12 drive cycles for the same vehicle. The results were then compared to the optimal results achieved by PMP, in which the optimization was done numerically. The fuel economy measures achieved by the proposed A-ECMS are very close to the ones found by PMP for all 12 drive cycles. This is partly due to the accuracy of the proposed analytical solution. More importantly, the AECMS results are attainable in real-world scenarios, unlike the PMP values that are not achievable due to imperfect driver model and non-causal nature of the method. For the considered drive cycles, the proposed A-ECMS is approximately 45 times faster than the standard ECMS on average, which can be further improved by adapting equivalence factor periodically instead of instantly. Finally, another A-ECMS method that is based on estimated equivalence factor bounds was implemented, and it was shown that non-inclusion of information about the ongoing drive cycle can adversely affect state of charge, causing it to operate far from acceptable SOC levels. In the proposed method, however, incorporation of nominal equivalence factors was observed to sustain electric charge for imperfect drive cycles.

\section{ACKNOWLEDGMENT}

The authors want to thank Swedish Energy Agency for funding the project, and also Volvo Construction Equipment for technical support.

\section{REFERENCES}

[1] A. A. Malikopoulos, "Supervisory power management control algorithms for hybrid electric vehicles: A survey," IEEE Transactions on intelligent transportation systems, vol. 15, no. 5, pp. 1869-1885, 2014.

[2] Y. Huang, H. Wang, A. Khajepour, H. He, and J. Ji, "Model predictive control power management strategies for hevs: A review," Journal of Power Sources, vol. 341, pp. 91-106, 2017.

[3] M. Sabri, K. Danapalasingam, and M. Rahmat, "A review on hybrid electric vehicles architecture and energy management strategies," Renewable and Sustainable Energy Reviews, vol. 53, pp. 1433-1442, 2016.

[4] L. V. Pérez, G. R. Bossio, D. Moitre, and G. O. García, "Optimization of power management in an hybrid electric vehicle using dynamic programming," Mathematics and Computers in Simulation, vol. 73, no. 1-4, pp. 244-254, 2006. 
[5] T. Nüesch, M. Wang, P. Isenegger, C. H. Onder, R. Steiner, P. MacriLassus, and L. Guzzella, "Optimal energy management for a diesel hybrid electric vehicle considering transient pm and quasi-static nox emissions," Control Engineering Practice, vol. 29, pp. 266-276, 2014.

[6] F. Zhang, L. Wang, S. Coskun, H. Pang, Y. Cui, and J. Xi, "Energy management strategies for hybrid electric vehicles: Review, classification, comparison, and outlook," Energies, vol. 13, no. 13, p. 3352, 2020.

[7] T. Leroy, F. Vidal-Naquet, and P. Tona, "Stochastic dynamic programming based energy management of hev's: an experimental validation," IFAC Proceedings Volumes, vol. 47, no. 3, pp. 4813-4818, 2014.

[8] T. Leroy, J. Malaizé, and G. Corde, "Towards real-time optimal energy management of hev powertrains using stochastic dynamic programming," in 2012 IEEE Vehicle Power and Propulsion Conference. IEEE, 2012, pp. 383-388.

[9] J. M. Luján, C. Guardiola, B. Pla, and A. Reig, "Analytical optimal solution to the energy management problem in series hybrid electric vehicles," IEEE Transactions on Vehicular Technology, vol. 67, no. 8 , pp. 6803-6813, 2018.

[10] S. Onori, L. Serrao, and G. Rizzoni, Hybrid electric vehicles: Energy management strategies. Springer, 2016.

[11] N. Kim, S. Cha, and H. Peng, "Optimal control of hybrid electric vehicles based on pontryagin's minimum principle," IEEE Transactions on control systems technology, vol. 19, no. 5, pp. 1279-1287, 2010.

[12] L. Serrao, S. Onori, and G. Rizzoni, "Ecms as a realization of pontryagin's minimum principle for hev control," in 2009 American control conference. IEEE, 2009, pp. 3964-3969.

[13] S. Onori, L. Serrao, and G. Rizzoni, "Adaptive equivalent consumption minimization strategy for hybrid electric vehicles," in ASME $2010 d y$ namic systems and control conference. American Society of Mechanical Engineers Digital Collection, 2010, pp. 499-505.

[14] S. Onori and L. Serrao, "On adaptive-ecms strategies for hybrid electric vehicles," in Proceedings of the international scientific conference on hybrid and electric vehicles, Malmaison, France, vol. 67, 2011.

[15] A. Chasse, A. Sciarretta, and J. Chauvin, "Online optimal control of a parallel hybrid with costate adaptation rule," IFAC proceedings volumes, vol. 43, no. 7, pp. 99-104, 2010.

[16] X. Tian, Y. Cai, X. Sun, Z. Zhu, and Y. Xu, "An adaptive ecms with driving style recognition for energy optimization of parallel hybrid electric buses," Energy, vol. 189, p. 116151, 2019.

[17] Y. Li and X. Jiao, "Real-time energy management for commute hevs using modified a-ecms with traffic information recognition," IET Intelligent Transport Systems, vol. 13, no. 4, pp. 729-737, 2018.

[18] S. Yang, W. Wang, F. Zhang, Y. Hu, and J. Xi, "Driving-style-oriented adaptive equivalent consumption minimization strategies for hevs," IEEE Transactions on Vehicular Technology, vol. 67, no. 10, pp. 9249-9261, 2018.

[19] X. Tian, R. He, X. Sun, Y. Cai, and Y. Xu, "An anfis-based ecms for energy optimization of parallel hybrid electric bus," IEEE Transactions on Vehicular Technology, 2019.

[20] C. Sun, F. Sun, and H. He, "Investigating adaptive-ecms with velocity forecast ability for hybrid electric vehicles," Applied Energy, vol. 185, pp. 1644-1653, 2017.

[21] D. Ambuhl and L. Guzzella, "Predictive reference signal generator for hybrid electric vehicles," IEEE transactions on vehicular technology, vol. 58, no. 9, pp. 4730-4740, 2009.

[22] F. Tianheng, Y. Lin, G. Qing, H. Yanqing, Y. Ting, and Y. Bin, "A supervisory control strategy for plug-in hybrid electric vehicles based on energy demand prediction and route preview," IEEE Transactions on Vehicular Technology, vol. 64, no. 5, pp. 1691-1700, 2014.

[23] J. T. Kessels, M. W. Koot, P. P. Van Den Bosch, and D. B. Kok, "Online energy management for hybrid electric vehicles," IEEE Transactions on vehicular technology, vol. 57, no. 6, pp. 3428-3440, 2008.

[24] A. Rezaei, J. B. Burl, and B. Zhou, "Estimation of the ecms equivalent factor bounds for hybrid electric vehicles," IEEE Transactions on Control Systems Technology, vol. 26, no. 6, pp. 2198-2205, 2017.

[25] A. Rezaei, J. B. Burl, B. Zhou, and M. Rezaei, "A new real-time optimal energy management strategy for parallel hybrid electric vehicles," IEEE Transactions on Control Systems Technology, vol. 27, no. 2, pp. 830837, 2017.

[26] M. Sivertsson, C. Sundström, and L. Eriksson, "Adaptive control of a hybrid powertrain with map-based ecms," IFAC Proceedings Volumes, vol. 44, no. 1, pp. 2949-2954, 2011

[27] A. E. Bryson, Applied optimal control: optimization, estimation and control. CRC Press, 1975.

[28] L. Guzzella, A. Sciarretta et al., Vehicle propulsion systems. Springer, 2007, vol. 1.
[29] L. Tang and G. Rizzoni, "Energy management strategy including battery life optimization for a hev with a cvt," in 2016 IEEE Transportation Electrification Conference and Expo, Asia-Pacific (ITEC Asia-Pacific), 2016, pp. 549-554.

[30] B. Frank, L. Skogh, and M. Alaküla, "On wheel loader fuel efficiency difference due to operator behaviour distribution," in 2nd International Commercial Vehicle Technology Symposium, CVT, 2012.

[31] R. Filla, "Operator and machine models for dynamic simulation of construction machinery," Ph.D. dissertation, Institutionen för konstruktionsoch produktionsteknik, 2005.

[32] S. Xie, H. Li, Z. Xin, T. Liu, and L. Wei, "A pontryagin minimum principle-based adaptive equivalent consumption minimum strategy for a plug-in hybrid electric bus on a fixed route," Energies, vol. 10, no. 9, p. 1379, 2017.

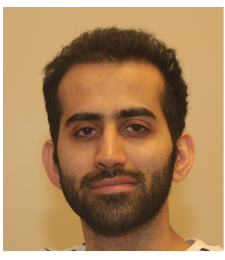

Iman Shafikhani received his bachelor's and master's degrees in electrical engineering, with focus on control theory, from University of Tehran (2012) and Tarbiat Modares University (2015), respectively. He is currently a Ph.D. student at the Department of Electrical Engineering at Linköping University. His research interests include optimal and model predictive control with applications in energy management of hybrid electric vehicles.

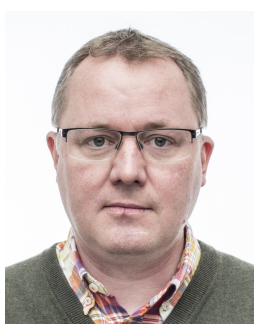

Jan Åslund was born in Boden, Sweden in 1971. $\mathrm{He}$ received a Ph.D. in Mathematics in 2002 from Linköping University, Sweden, and currently he has a position as Associate Professor at the Department of Electrical Engineering at Linköping University. His research interests include model based diagnosis and optimal control. 